TRANSACTIONS OF THE

AMERICAN MATHEMATICAL SOCIETY

Volume 356, Number 7, Pages 2709-2737

S 0002-9947(03)03332-4

Article electronically published on October 21, 2003

\title{
FUNDAMENTAL SOLUTIONS FOR NON-DIVERGENCE FORM OPERATORS ON STRATIFIED GROUPS
}

\author{
ANDREA BONFIGLIOLI, ERMANNO LANCONELLI, AND FRANCESCO UGUZZONI
}

\begin{abstract}
We construct the fundamental solutions $\Gamma$ and $\gamma$ for the nondivergence form operators $\sum_{i, j} a_{i, j}(x, t) X_{i} X_{j}-\partial_{t}$ and $\sum_{i, j} a_{i, j}(x) X_{i} X_{j}$, where the $X_{i}$ 's are Hörmander vector fields generating a stratified group $\mathbb{G}$ and $\left(a_{i, j}\right)_{i, j}$ is a positive-definite matrix with Hölder continuous entries. We also provide Gaussian estimates of $\Gamma$ and its derivatives and some results for the relevant Cauchy problem. Suitable long-time estimates of $\Gamma$ allow us to construct $\gamma$ using both $t$-saturation and approximation arguments.
\end{abstract}

\section{INTRODUCTION AND MAIN RESULTS}

The aim of this paper is to prove the existence and some regularity properties of the fundamental solution for the following non-divergence form operators:

$$
\mathcal{H}=\sum_{i, j=1}^{m} a_{i, j}(x, t) X_{i} X_{j}-\partial_{t}, \quad \mathcal{L}=\sum_{i, j=1}^{m} a_{i, j}(x) X_{i} X_{j} .
$$

Here $\left\{X_{i}\right\}_{i}$ is a stratified system of vector fields in $\mathbb{R}^{N}$ and $\left(a_{i, j}\right)_{i, j \leq m}$ is a positivedefinite matrix with Hölder continuous entries.

Our main results are contained in Theorem 1.2 and Theorem 1.5 below. As a byproduct we also give an existence theorem for the Cauchy problem related to $\mathcal{H}$ (see Theorem [1.2.(iv) and also Theorem [3.1). The approach we follow in constructing the fundamental solution $\Gamma$ for $\mathcal{H}$ is based on an adaptation of Levi's classical parametrix method. Crucial requirements for our procedure are uniform Gaussian estimates of the fundamental solutions $\Gamma_{z_{0}}$ of the "constant coefficient" operators

$$
\mathcal{H}_{z_{0}}=\sum_{i, j=1}^{m} a_{i, j}\left(z_{0}\right) X_{i} X_{j}-\partial_{t}
$$

obtained by freezing $\mathcal{H}$ at $z_{0} \in \mathbb{R}^{N+1}$. These non-trivial estimates have been recently proved in [2] and are based on a study started in [3, 4]. For reading convenience we recall them in Theorem 1.1. We would like to emphasize that the lack of knowledge of an explicit expression of the parametrix $\Gamma_{z_{0}}$ makes Levi's method more involved in our context than in the classical one.

Received by the editors November 21, 2002.

2000 Mathematics Subject Classification. Primary 35A08, 35H20, 43A80; Secondary 35A17, 35J70.

Key words and phrases. Non-divergence sub-elliptic operators, stratified groups, fundamental solutions.

Investigation supported by University of Bologna, Funds for selected research topics.

(C)2003 American Mathematical Society 
By integrating $\Gamma$ over the time variable $t$, we are able to construct the fundamental solution $\gamma$ for the operator $\mathcal{L}$. This can be done provided suitable long-time estimates of $\Gamma$ are established. On the other hand, whereas optimal small-time estimates of $\Gamma$ (and of its derivatives) can be directly obtained from its construction (see Theorem 1.2-(ii)), proving long-time estimates is a more delicate matter. We are able to obtain the latter up to modifying the coefficients $a_{i, j}$ 's outside a compact set (see Theorem 1.4). We can then obtain a local fundamental solution for $\mathcal{L}$ using both $t$-saturation and approximation arguments. We would like to observe that this procedure is not straightforward; to the authors' knowledge, only a few results are present in the literature even in the case of fundamental solutions for classical stationary operators $\mathcal{L}=\sum_{i, j} a_{i, j}(x) \partial_{i} \partial_{j}$ (see, e.g., 25, 35]). The classical parabolic case is treated in detail in the monograph [19.

Before proceeding, we would like to give some motivation for our research. In recent years there has been a growing interest in the study of second order linear and nonlinear partial differential equations of subelliptic type. These equations arise in many different settings: geometric theory of several complex variables, curvature problems for CR-manifolds, sub-Riemannian geometry, diffusion processes, control theory, human vision; see, e.g., [15, 16, 22, 23, 28, 29, 34, 36, 37, 40, 41, 43]. The common features of such equations are the following ones: their characteristic forms are non-negative definite and their ellipticity directions span at any point subspaces of dimension strictly less than that of the space. The complementary directions are recovered by commutations.

It follows that the underlying algebraic structures of all these equations are homogeneous Carnot groups $\mathbb{G}$, i.e., stratified Lie groups on $\mathbb{R}^{N}$ (see the definition below). In these contexts, the basic subelliptic operators of order two take the divergence form

$$
-\sum_{i, j=1}^{m} X_{i}^{*}\left(a_{i, j} X_{j}\right)
$$

or the non-divergence form

$$
\mathcal{L}=\sum_{i, j=1}^{m} a_{i, j} X_{i} X_{j}
$$

where $\left\{X_{i}\right\}_{i}$ is a basis of the first layer of the stratification of $\mathfrak{g}$, the Lie algebra of G. $X_{i}^{*}$ denotes the adjoint of $X_{i}$ and $\left(a_{i, j}\right)_{i, j \leq m}$ is a positive-definite $m \times m$ matrix.

The parabolic counterpart of the operators in (1.3) and (1.4) are also of great relevance; see, e.g., [1. 43. We would also like to mention the papers by Huisken and Klingenberg [22] and Montanari [34, in which operators like the first one in (1.11) arise in studying motion by Levi-curvature of hypersurfaces of $\mathbb{C}^{n+1}$.

Several results concerning divergence form operators (1.3) are present in the literature, both for linear and quasi-linear equations. Harnack's inequality, regularity results for solutions, and existence and size estimates of the Green's function can be found, e.g., in the papers [9, 10, 11, 12, 14, 18, 30, 31, 32, 33, 44; ; see also the references therein. On the contrary, to the authors' knowledge, very few papers are devoted to non-divergence form operators. We may just quote some works by Bramanti and Brandolini [7, 8, by Xu [44, and the recent paper by Capogna and Han [13], where a priori estimates in $L^{p}$ and Hölder spaces are proved. We also quote a paper by Polidoro [38], where the Levi parametrix method is applied to construct the fundamental solutions for ultraparabolic equations of Kolmogorov type.

Our aim in this paper is to provide a new contribution to the study of nondivergence form operators (1.4). 
We now introduce some notation and then state our main results. We start by giving the definition of a Carnot group. Let $\circ$ be an assigned Lie group law on $\mathbb{R}^{N}$. Suppose $\mathbb{R}^{N}$ is endowed with a homogeneous structure by a given family of Lie group automorphisms $\left\{\delta_{\lambda}\right\}_{\lambda>0}$ (called dilations) of the form $\delta_{\lambda}(x)=$ $\delta_{\lambda}\left(x^{(1)}, x^{(2)}, \ldots, x^{(r)}\right)=\left(\lambda x^{(1)}, \lambda^{2} x^{(2)}, \ldots, \lambda^{r} x^{(r)}\right)$. Here $x^{(i)} \in \mathbb{R}^{N_{i}}$ for $i=1, \ldots, r$ and $N_{1}+\cdots+N_{r}=N$. We denote by $\mathfrak{g}$ the Lie algebra of $\left(\mathbb{R}^{N}, \circ\right)$, i.e., the Lie algebra of left-invariant vector fields on $\mathbb{R}^{N}$. For $i=1, \ldots, N_{1}$, let $X_{i}$ be the (unique) vector field in $\mathfrak{g}$ that agrees at the origin with $\partial / \partial x_{i}^{(1)}$. We make the following assumption: the Lie algebra generated by $X_{1}, \ldots, X_{N_{1}}$ is the whole $\mathfrak{g}$. With the above hypotheses, we call $\mathbb{G}=\left(\mathbb{R}^{N}, \circ, \delta_{\lambda}\right)$ a homogeneous Carnot group. We also say that $\mathbb{G}$ is of step $r$ and has $m:=N_{1}$ generators. The canonical sub-Laplacian on $\mathbb{G}$ is the second order differential operator $\Delta_{\mathbb{G}}=\sum_{i=1}^{m} X_{i}^{2}$. If $Y_{1}, \ldots, Y_{m}$ is any basis for $\operatorname{span}\left\{X_{1}, \ldots, X_{m}\right\}$, the second order differential operator $\sum_{i=1}^{m} Y_{i}^{2}$ will be called a sub-Laplacian on $\mathbb{G}$. In the literature (see, e.g., [17, 20, 39, 43]) a Carnot group (or stratified group) $\mathbb{H}$ is defined as a connected and simply connected Lie group whose Lie algebra $\mathfrak{h}$ admits a stratification $\mathfrak{h}=\mathfrak{G}_{1} \oplus \cdots \oplus \mathfrak{G}_{r}$ with $\left[\mathfrak{G}_{1}, \mathfrak{G}_{i}\right]=\mathfrak{G}_{i+1}$, $\left[\mathfrak{G}_{1}, \mathfrak{G}_{r}\right]=\{0\}$. It is not difficult to recognize that any homogeneous Carnot group is a Carnot group according to the classical definition. On the other hand, up to isomorphism, the opposite implication is also true (see, e.g., 4]). We denote by $Q=\sum_{j=1}^{r} j N_{j}$ the homogeneous dimension of $\mathbb{G}$. If $Q \leq 3$, then $\mathbb{G}$ is the ordinary Euclidean group $\left(\mathbb{R}^{Q},+\right)$ and $\Delta_{\mathbb{G}}$ is the classical Laplace operator. Hence, throughout the paper, we shall always assume $Q \geq 4$. In the sequel, $d$ will denote a fixed homogeneous norm on $\mathbb{G}$, i.e., a continuous function $d: \mathbb{R}^{N} \rightarrow[0, \infty)$, smooth away from the origin, such that $d\left(\delta_{\lambda}(x)\right)=\lambda d(x), d\left(x^{-1}\right)=d(x)$, and $d(x)=0$ iff $x=0$. Hereafter, we also denote $d\left(y^{-1} \circ x\right)$ by $d(x, y)$ and use the notation $B_{d}(x, r)$ for the $d$-ball of center $x \in \mathbb{G}$ and radius $r>0$. The following quasi-triangle inequality holds for a suitable constant $c$ :

$$
d(x, y) \leq c(d(x, z)+d(z, y))
$$

Given $\Lambda>1$, we shall denote by $\mathcal{M}_{\Lambda}$ the class of the real $m \times m$ symmetric matrices $A$ such that $\Lambda^{-1}|\xi|^{2} \leq\langle A \xi, \xi\rangle \leq \Lambda|\xi|^{2}$, for every $\xi \in \mathbb{R}^{m}$. For $A \in \mathcal{M}_{\Lambda}$, we denote by $\mathcal{H}_{A}$ the constant coefficient $X$-parabolic operator on $\mathbb{G} \times \mathbb{R} \equiv \mathbb{R}^{N+1}$,

$$
\mathcal{H}_{A}=\mathcal{L}_{A}-\partial_{t}=\sum_{i, j=1}^{m} a_{i, j} X_{i} X_{j}-\partial_{t}
$$

Hereafter, we use the notation $z=(x, t)$ for points of $\mathbb{R}^{N+1}(x \in \mathbb{G}, t \in \mathbb{R})$. We now briefly recall some well-known results on the fundamental solution for $\mathcal{H}_{A}$. There exists a non-negative smooth function $\Gamma_{A}$ on $\mathbb{R}^{N+1} \backslash\{0\}$ such that the fundamental solution for $\mathcal{H}_{A}$ is given by

$$
\Gamma_{A}(x, t ; \xi, \tau):=\Gamma_{A}\left(\xi^{-1} \circ x, t-\tau\right) .
$$

It is $\Gamma_{A}(x, t)=0$ iff $t \leq 0, \Gamma_{A}(x, t)=\Gamma_{A}\left(x^{-1}, t\right)$ and $\Gamma_{A}\left(\delta_{\lambda}(x), \lambda^{2} t\right)=\lambda^{-Q} \Gamma_{A}(x, t)$; in particular, $\Gamma_{A}$ vanishes at infinity. For every $\zeta \in \mathbb{R}^{N+1}, \Gamma_{A}(\cdot ; \zeta)$ is locally integrable and $\mathcal{H}_{A} \Gamma_{A}(\cdot, \zeta)=-\delta_{\zeta}$ (the Dirac measure supported at $\{\zeta\}$ ). For every $t>0$, we have

$$
\int_{\mathbb{R}^{N}} \Gamma_{A}(x, t) d x=1 .
$$


For every $x \in \mathbb{R}^{N}, t>0$ and $\tau>0$, the following reproduction property holds:

$$
\Gamma_{A}(x, t+\tau)=\int_{\mathbb{R}^{N}} \Gamma_{A}\left(\xi^{-1} \circ x, t\right) \Gamma_{A}(\xi, \tau) d \xi .
$$

One of the main tools we shall use in this paper is the following theorem, proved in 2], where we establish uniform Gaussian estimates for $\Gamma_{A}$ when $A$ varies in the class $\mathcal{M}_{\Lambda}$.

Theorem 1.1. Given any non-negative integers $p, q$, there exist positive constants $c_{\Lambda}, c_{\Lambda, p}, c_{\Lambda, p, q}$ such that for every $i_{1}, \ldots, i_{p} \in\{1, \ldots, m\}$ and for every $A, A_{1}, A_{2} \in$ $\mathcal{M}_{\Lambda}$, we have

$$
\begin{gathered}
c_{\Lambda}^{-1} t^{-Q / 2} \exp \left(-\frac{c_{\Lambda} d^{2}(x)}{t}\right) \leq \Gamma_{A}(x, t) \leq c_{\Lambda} t^{-Q / 2} \exp \left(-\frac{d^{2}(x)}{c_{\Lambda} t}\right), \\
\left|X_{i_{1}} \cdots X_{i_{p}}\left(\partial_{t}\right)^{q} \Gamma_{A}(x, t)\right| \leq c_{\Lambda, p, q} t^{-(Q+p+2 q) / 2} \exp \left(-\frac{d^{2}(x)}{c_{\Lambda} t}\right), \\
\left|X_{i_{1}} \cdots X_{i_{p}}\left(\partial_{t}\right)^{q} \Gamma_{A_{1}}(x, t)-X_{i_{1}} \cdots X_{i_{p}}\left(\partial_{t}\right)^{q} \Gamma_{A_{2}}(x, t)\right| \\
\leq c_{\Lambda, p, q}\left\|A_{1}-A_{2}\right\|^{1 / r} t^{-(Q+p+2 q) / 2} \exp \left(-\frac{d^{2}(x)}{c_{\Lambda} t}\right),
\end{gathered}
$$

for every $x \in \mathbb{R}^{N}, t>0$. Here, $\|A\|$ denotes the matrix norm $\max _{|\xi|=1}|A \xi|$. We also recall that $r$ denotes the step of $\mathbb{G}$.

In order to state our main results, Theorems 1.2 and 1.5, we now introduce the following regularity classes of functions. Given an arbitrary domain $\Omega \subseteq \mathbb{R}^{N}$, we denote by $\Gamma^{\beta}(\Omega)(0<\beta<1)$ the space of functions $v: \Omega \rightarrow \mathbb{R}$ such that

$$
\|v\|_{\Gamma^{\beta}(\Omega)}:=\sup _{\Omega}|v|+\sup _{x \neq x^{\prime} \in \Omega} \frac{\left|v(x)-v\left(x^{\prime}\right)\right|}{d\left(x, x^{\prime}\right)^{\beta}}<\infty .
$$

Moreover, we denote by $\Gamma^{2+\beta}(\Omega)$ the space of functions $v$ which belong to $\Gamma^{\beta}(\Omega)$ together with any Lie derivative along the vector fields $X_{1}, \ldots, X_{m}$ up to second order. We also denote by $\Gamma_{\mathrm{loc}}^{2+\beta}(\Omega)$ the space of functions $v: \Omega \rightarrow \mathbb{R}$ such that $v$ and its Lie derivatives along the vector fields $X_{1}, \ldots, X_{m}$ up to second order satisfy (1.7) with $\Omega$ replaced by $\bar{O}$, for every bounded domain $O \subset \bar{O} \subset \Omega$. If $\Omega$ is an arbitrary domain of $\mathbb{R}^{N+1}$, we shall use the same notation $\Gamma^{\beta}(\Omega)$ to denote the space of functions $u: \Omega \rightarrow \mathbb{R}$ such that

$$
\|u\|_{\Gamma^{\beta}(\Omega)}:=\sup _{\Omega}|u|+\sup _{(x, t) \neq\left(x^{\prime}, t^{\prime}\right) \in \Omega} \frac{\left|u(x, t)-u\left(x^{\prime}, t^{\prime}\right)\right|}{d\left(x, x^{\prime}\right)^{\beta}+\left|t-t^{\prime}\right|^{\beta / 2}}<\infty .
$$

Moreover, $\Gamma^{2+\beta}(\Omega)$ will denote the space of functions $u: \Omega \rightarrow \mathbb{R}$ which belong to $\Gamma^{\beta}(\Omega)$ together with any Lie derivative along the vector fields $X_{1}, \ldots, X_{m}$ up to second order, and along $\partial_{t}$ up to first order. Analogously one defines $\Gamma_{\operatorname{loc}}^{2+\beta}(\Omega)$.

Theorem 1.2. Consider the operator $\mathcal{H}=\sum_{i, j=1}^{m} a_{i, j}(x, t) X_{i} X_{j}-\partial_{t}$, with coefficients $a_{i, j}$ in the Hölder space $\Gamma^{\alpha}\left(\mathbb{R}^{N+1}\right)$ and in the class $\mathcal{M}_{\Lambda}$. Then there exists a fundamental solution $\Gamma$ for $\mathcal{H}$, with the properties listed below.

(i) $\Gamma$ is a continuous function away from the diagonal of $\mathbb{R}^{N+1} \times \mathbb{R}^{N+1}$. Moreover, for every fixed $\zeta \in \mathbb{R}^{N+1}, \Gamma(\cdot ; \zeta) \in \Gamma_{\mathrm{loc}}^{2+\alpha}\left(\mathbb{R}^{N+1} \backslash\{\zeta\}\right)$, and we have

$$
\mathcal{H}(\Gamma(\cdot ; \zeta))=0 \quad \text { in } \mathbb{R}^{N+1} \backslash\{\zeta\} .
$$


(ii) $\Gamma(x, t ; \xi, \tau)=0$ for $t \leq \tau$. Moreover, there exist a positive constant $M$ and, for every $T>0$, a positive constant $c(T)$ such that, for $0<t-\tau \leq T$, the following estimates hold:

$$
\begin{gathered}
0 \leq \Gamma(x, t ; \xi, \tau) \leq c(T)(t-\tau)^{-Q / 2} \exp \left(-\frac{d^{2}(x, \xi)}{M(t-\tau)}\right), \\
\left|X_{j}(\Gamma(\cdot ; \xi, \tau))(x, t)\right| \leq c(T)(t-\tau)^{-(Q+1) / 2} \exp \left(-\frac{d^{2}(x, \xi)}{M(t-\tau)}\right), \\
\left|X_{i} X_{j}(\Gamma(\cdot ; \xi, \tau))(x, t)\right|,\left|\partial_{t}(\Gamma(\cdot ; \xi, \tau))(x, t)\right| \\
\leq c(T)(t-\tau)^{-(Q+2) / 2} \exp \left(-\frac{d^{2}(x, \xi)}{M(t-\tau)}\right) .
\end{gathered}
$$

(iii) For every $\psi \in C_{0}^{\infty}\left(\mathbb{R}^{N+1}\right)$, the function

$$
w(z)=\int_{\mathbb{R}^{N+1}} \Gamma(z ; \zeta) \psi(\zeta) d \zeta
$$

belongs to the class $\Gamma_{\mathrm{loc}}^{2+\alpha}\left(\mathbb{R}^{N+1}\right)$, and we have

$$
\mathcal{H} w=-\psi \quad \text { in } \mathbb{R}^{N+1} .
$$

(iv) Let $\mu \geq 0$ and $T_{2}>T_{1}$ be such that $\left(T_{2}-T_{1}\right) \mu$ is small enough. Then, for every $f \in \Gamma^{\beta}\left(\mathbb{R}^{N} \times\left[T_{1}, T_{2}\right]\right.$ ) (where $0<\beta \leq \alpha$ ) and $g \in C\left(\mathbb{R}^{N}\right)$ satisfying the growth condition $|f(x, t)|,|g(x)| \leq c \exp \left(\mu d^{2}(x)\right)$ for some constant $c>0$, the function

$$
\begin{gathered}
u(x, t)=\int_{\mathbb{R}^{N}} \Gamma\left(x, t ; \xi, T_{1}\right) g(\xi) d \xi+\int_{\mathbb{R}^{N} \times\left[T_{1}, t\right]} \Gamma(x, t ; \xi, \tau) f(\xi, \tau) d \xi d \tau \\
\left(x \in \mathbb{R}^{N}, t \in\left(T_{1}, T_{2}\right]\right) \text { belongs to the class } \\
\Gamma_{\text {loc }}^{2+\beta}\left(\mathbb{R}^{N} \times\left(T_{1}, T_{2}\right)\right) \cap C\left(\mathbb{R}^{N} \times\left[T_{1}, T_{2}\right]\right) .
\end{gathered}
$$

Moreover, $u$ is a solution to the following Cauchy problem:

$$
\left\{\begin{array}{l}
\mathcal{H} u=-f \quad \text { in } \mathbb{R}^{N} \times\left(T_{1}, T_{2}\right), \\
u\left(\cdot, T_{1}\right)=g .
\end{array}\right.
$$

Remark 1.3. We refer to Theorem 3.1 for a slightly stronger version of (iv).

Starting from Theorem 1.2, one can construct local fundamental solutions for the $X$-elliptic operator $\mathcal{L}=\sum_{i, j=1}^{m} a_{i, j}(x) X_{i} X_{j}$, by integrating $\Gamma$ over the variable $t$, provided suitable long-time estimates of $\Gamma$ can be established. We are able to find the needed long-time estimates if the dimension $m$ of the first layer of the stratification of $\mathfrak{g}$ is strictly greater than two. Indeed, we can prove the following theorem.

Theorem 1.4. Let $\Omega$ be a bounded domain of $\mathbb{R}^{N+1}$ and let $\mathcal{H}$ be the differential operator in Theorem 1.2. Up to modifying the coefficients $a_{i, j}(x, t)$ of $\mathcal{H}$ outside $\Omega$, the fundamental solution $\Gamma$ for $\mathcal{H}$ satisfies the following estimate: for every compact set $K \Subset \mathbb{R}^{m}$, there exist positive constants $c, M$ such that

$$
\Gamma(x, t ; \xi, \tau) \leq c(M+t-\tau)^{-m / 2} \exp \left(-\frac{\Lambda\left|x^{(1)}-\xi^{(1)}\right|^{2}}{M+t-\tau}\right),
$$

for every $(x, t),(\xi, \tau) \in \mathbb{R}^{N+1}$ such that $t>\tau+1$ and $\xi^{(1)} \in K$. We recall that $x^{(1)}$ denotes the vector of the first $m$ coordinates of $x$. 
The estimate (1.8) is certainly not optimal, but it is indeed sufficient to ensure the convergence of the integral $\int_{\mathbb{R}} \Gamma(x, t ; \xi, 0) d t$ (for $x \neq \xi$ ) if $m>2$. This allows us to obtain the following result.

Theorem 1.5. Suppose that $m>2$. Let us consider the differential operator $\mathcal{L}=\sum_{i, j=1}^{m} a_{i, j}(x) X_{i} X_{j}$, with coefficients $a_{i, j}$ in the Hölder space $\Gamma^{\alpha}\left(\mathbb{R}^{N}\right)$ and in the class $\mathcal{M}_{\Lambda}$. For every fixed bounded open set $\Omega \subset \mathbb{R}^{N}$, there exists a function $\gamma$ defined on $\mathbb{R}^{N} \times \mathbb{R}^{N}$ which is a fundamental solution for $\mathcal{L}$ in $\Omega$ (in the sense below), with the following properties.

(i) $\gamma$ is a continuous function away from the diagonal of $\mathbb{R}^{N} \times \mathbb{R}^{N}$. Moreover, for every fixed $\xi \in \mathbb{R}^{N}, \gamma(\cdot, \xi) \in \Gamma_{\text {loc }}^{2+\alpha}\left(\mathbb{R}^{N} \backslash\{\xi\}\right)$, and we have

$$
\mathcal{L}(\gamma(\cdot, \xi))=0 \quad \text { in } \Omega \backslash\{\xi\} .
$$

(ii) For every compact set $K \Subset \mathbb{R}^{N}$, there exists a positive constant $c$ such that

$$
0 \leq \gamma(x, \xi) \leq c\left(1+d(x, \xi)^{2-Q}\right), \quad \xi \in K, x \in \mathbb{R}^{N} .
$$

(iii) For every $\psi \in C_{0}^{\infty}\left(\mathbb{R}^{N}\right)$, the function

$$
w(x)=\int_{\mathbb{R}^{N}} \gamma(x ; \xi) \psi(\xi) d \xi
$$

belongs to the class $\Gamma_{\mathrm{loc}}^{2+\alpha}\left(\mathbb{R}^{N}\right)$, and we have

$$
\mathcal{L} w=-\psi \quad \text { in } \Omega .
$$

Remark 1.6. The function $\gamma$ in Theorem 1.5 is indeed a global fundamental solution of an operator $\widetilde{\mathcal{L}}$ (agreeing with $\mathcal{L}$ in $\Omega$ ) obtained from $\mathcal{L}$ up to suitably modifying the coefficients $a_{i, j}$ outside $\Omega$ (see Theorem [5.2).

The paper is organized as follows. In Section 2 we construct the fundamental solution $\Gamma$ for $\mathcal{H}$, providing Gaussian estimates of $\Gamma$ and its derivatives. We also introduce the "weak" class of regularity $\mathfrak{C}^{2}$, and we show that $\Gamma$ belongs to this class. In Section 3 the Cauchy problem for $\mathcal{H}$ is studied. In Section 4 we prove the long-time estimates of $\Gamma$ of Theorem 1.4. To this end, we prove a weak maximum principle for $\mathcal{H}$ in the class $\mathfrak{C}^{2}$. We then complete the proof of Theorem 1.2 Finally, in Section 5 we prove Theorem 1.5, constructing the fundamental solution $\gamma$ for $\mathcal{L}$.

We conclude this introduction by observing that also a Gaussian estimate of $\Gamma$ from below holds. This estimate is also related to a Harnack inequality for $\mathcal{H}$ of Krylov-Safonov type. However, the generalization of such a Harnack inequality to our context seems to be non-trivial. This topic is investigated in a forthcoming paper (A. Bonfiglioli and F. Uguzzoni, Harnack inequality for non-divergence form operators on stratified groups, preprint).

\section{Construction of the fundamental solution for $\mathcal{H}$}

Let us fix the parameters $\Lambda>1, k>0$ and $\alpha \in(0,1]$. Throughout the paper, we shall denote by $\mathbf{c}$ any positive constant that depends only on these parameters (and on the structure of $\mathbb{G}$ ). Moreover, we shall use the notation $\mathbf{c}\left(f_{1}, \ldots, f_{n}\right)$ if $\mathbf{c}$ also depends on $f_{1}, \ldots, f_{n}$. Let $A=\left(a_{i, j}\right)_{i, j \leq m}$ be a matrix-valued function defined on $\mathbb{R}^{N+1}$ such that

$$
A(x, t) \in \mathcal{M}_{\Lambda}, \quad\left|a_{i, j}(x, t)-a_{i, j}\left(x^{\prime}, t^{\prime}\right)\right| \leq k\left(d\left(x, x^{\prime}\right)^{\alpha}+\left|t-t^{\prime}\right|^{\alpha / 2}\right),
$$


for every $(x, t),\left(x^{\prime}, t^{\prime}\right) \in \mathbb{R}^{N+1}$. We denote by $\mathcal{H}$ the parabolic-type operator with variable coefficients

$$
\mathcal{H}=\mathcal{L}-\partial_{t}=\sum_{i, j=1}^{m} a_{i, j}(x, t) X_{i} X_{j}-\partial_{t}
$$

and (with a slightly different notation with respect to $\mathcal{H}_{A\left(\zeta_{0}\right)}$ of Section 1) by

$$
\mathcal{H}_{\zeta_{0}}=\mathcal{L}_{\zeta_{0}}-\partial_{t}=\sum_{i, j=1}^{m} a_{i, j}\left(\zeta_{0}\right) X_{i} X_{j}-\partial_{t},
$$

the related frozen operator at $\zeta_{0} \in \mathbb{R}^{N+1}$. From Theorem 1.1 and from (2.1), we know that the fundamental solutions $\Gamma_{\zeta_{0}}$ of $\mathcal{H}_{\zeta_{0}}$ (henceforth, we shall use this notation instead of $\Gamma_{A\left(\zeta_{0}\right)}$ of Section [1) satisfy the following estimates:

$$
\begin{gathered}
\mathbf{c}^{-1} \mathbf{E}\left(x, \mathbf{c}^{-1} t\right) \leq \Gamma_{\zeta_{0}}(x, t) \leq \mathbf{c} \mathbf{E}(x, \mathbf{c} t) \\
\left|X_{i_{1}} \cdots X_{i_{p}}\left(\partial_{t}\right)^{q} \Gamma_{\zeta_{0}}(x, t)\right| \leq \mathbf{c}(p, q) t^{-(p+2 q) / 2} \mathbf{E}(x, \mathbf{c} t) \\
\left|X_{i_{1}} \cdots X_{i_{p}}\left(\partial_{t}\right)^{q} \Gamma_{\zeta_{0}}(x, t)-X_{i_{1}} \cdots X_{i_{p}}\left(\partial_{t}\right)^{q} \Gamma_{\zeta_{1}}(x, t)\right| \\
\leq \mathbf{c}(p, q)\left(d\left(\xi_{0}, \xi_{1}\right)^{\alpha / r}+\left|\tau_{0}-\tau_{1}\right|^{\alpha /(2 r)}\right) t^{-(p+2 q) / 2} \mathbf{E}(x, \mathbf{c} t),
\end{gathered}
$$

for every $\zeta_{0}=\left(\xi_{0}, \tau_{0}\right), \zeta_{1}=\left(\xi_{1}, \tau_{1}\right) \in \mathbb{R}^{N+1}, x \in \mathbb{R}^{N}, t>0$. We have denoted, for simplicity of notation,

$$
\mathbf{E}(x, t)=t^{-Q / 2} \exp \left(-\frac{d(x)^{2}}{t}\right), \quad x \in \mathbb{R}^{N}, t>0 .
$$

We now set

$$
Z_{1}(z ; \zeta)=\mathcal{H}\left(z \mapsto \Gamma_{\zeta}\left(\xi^{-1} \circ x, t-\tau\right)\right)(z), \quad(x, t)=z \neq \zeta=(\xi, \tau) \in \mathbb{R}^{N+1} .
$$

Since $\mathcal{H}=\mathcal{L}-\mathcal{L}_{\zeta}+\mathcal{H}_{\zeta}$, we have

$$
Z_{1}(z ; \zeta)=\sum_{i, j=1}^{m}\left(a_{i, j}(z)-a_{i, j}(\zeta)\right) X_{i} X_{j} \Gamma_{\zeta}\left(\xi^{-1} \circ x, t-\tau\right) .
$$

Moreover, by (2.1), (2.2) and (2.3), we get

$$
\begin{aligned}
\left|Z_{1}(z ; \zeta)\right| & \leq \mathbf{c}(t-\tau)^{\frac{\alpha}{2}-1}\left(1+\frac{d\left(\xi^{-1} \circ x\right)^{\alpha}}{(t-\tau)^{\frac{\alpha}{2}}}\right) \mathbf{E}\left(\xi^{-1} \circ x, \mathbf{c}(t-\tau)\right) \\
& \leq \mathbf{c}(t-\tau)^{\frac{\alpha}{2}-1} \mathbf{E}\left(\xi^{-1} \circ x, \mathbf{c}(t-\tau)\right) \\
& \leq \mathbf{c}(t-\tau)^{\frac{\alpha}{2}-1} \Gamma_{\zeta_{0}}\left(\xi^{-1} \circ x, \mathbf{c}(t-\tau)\right),
\end{aligned}
$$

for every $\zeta_{0} \in \mathbb{R}^{N+1}$. Here we have also used the following property of $\mathbf{E}$ :

$$
\left(d(x)^{2} / t\right)^{\mu} \mathbf{E}(x, \lambda t) \leq c_{\mu} \lambda^{\mu} \mathbf{E}(x, 2 \lambda t), \quad \forall \lambda>0, \mu \geq 0 .
$$

Proposition 2.1. For every $j \in \mathbb{N}$,

$$
Z_{j+1}(z ; \zeta)=\int_{\mathbb{R}^{N} \times[\tau, t]} Z_{1}(z ; \eta) Z_{j}(\eta ; \zeta) d \eta, \quad z=(x, t), \zeta=(\xi, \tau) \in \mathbb{R}^{N+1}, t>\tau,
$$

is well posed by induction, and the following estimate holds (for every $\zeta_{0} \in \mathbb{R}^{N+1}$ )

$$
\left|Z_{j}(z ; \zeta)\right| \leq \mathbf{c}_{1}^{j} b_{j}(\alpha)(t-\tau)^{-1+j \alpha / 2} \Gamma_{\zeta_{0}}\left(\xi^{-1} \circ x, \mathbf{c}_{2}(t-\tau)\right),
$$

where $b_{j}(\alpha)=\Gamma^{j}\left(\frac{\alpha}{2}\right) / \Gamma\left(\frac{j \alpha}{2}\right)$ (here only, $\Gamma$ denotes the Euler gamma function). As a consequence, the series

$$
\Phi(z ; \zeta)=\sum_{j=1}^{\infty} Z_{j}(z ; \zeta)
$$


totally converges on the set $\left\{0<t-\tau \leq T, d(x, \xi)+|t-\tau|^{\frac{1}{2}} \geq 1 / T\right\}$ (for every $T>0)$, and satisfies the estimate

$$
|\Phi(z ; \zeta)| \leq \mathbf{c}(T)(t-\tau)^{\frac{\alpha}{2}-1} \mathbf{E}\left(\xi^{-1} \circ x, \mathbf{c}(t-\tau)\right), \quad 0<t-\tau \leq T .
$$

Proof. For $j=1$, (2.9) is given by (2.7). Assuming by induction that (2.9) holds for a given $j \in \mathbb{N}$, we get

$$
\begin{aligned}
& \int_{\mathbb{R}^{N} \times[\tau, t]}\left|Z_{1}(z ; \eta) Z_{j}(\eta ; \zeta)\right| d \eta \leq \mathbf{c}_{1}^{j+1} b_{j}(\alpha) \int_{\tau}^{t}(t-s)^{-1+\frac{\alpha}{2}}(s-\tau)^{-1+\frac{j \alpha}{2}} \\
& \times \int_{\mathbb{R}^{N}} \Gamma_{\zeta_{0}}\left(y^{-1} \circ x, \mathbf{c}_{2}(t-s)\right) \Gamma_{\zeta_{0}}\left(\xi^{-1} \circ y, \mathbf{c}_{2}(s-\tau)\right) d y d s \\
& =\mathbf{c}_{1}^{j+1} b_{j}(\alpha) \Gamma_{\zeta_{0}}\left(\xi^{-1} \circ x, \mathbf{c}_{2}(t-\tau)\right)(t-\tau)^{-1+\frac{(j+1) \alpha}{2}} \int_{0}^{1}(1-r)^{-1+\frac{\alpha}{2}} r^{-1+\frac{j \alpha}{2}} d r .
\end{aligned}
$$

In the last equality, we have used the reproduction property (1.6) of $\Gamma_{\zeta_{0}}$. Recalling the definition of $b_{j}(\alpha)$, we obtain (2.9) for $j+1$. In order to complete the proof, it is now sufficient to observe that the power series $\sum_{j=1}^{\infty} b_{j}(\alpha) w^{j}$ has infinite radius of convergence.

Corollary 2.2. We have

$$
\Phi(z ; \zeta)=Z_{1}(z ; \zeta)+\int_{\mathbb{R}^{N} \times[\tau, t]} Z_{1}(z ; \eta) \Phi(\eta ; \zeta) d \eta,
$$

for every $z=(x, t), \zeta=(\xi, \tau) \in \mathbb{R}^{N+1}, t>\tau$.

Proof. From the proof of Proposition 2.1, it follows that the series

$$
\sum_{j=1}^{\infty} \int_{\mathbb{R}^{N} \times[\tau, t]}\left|Z_{1}(z ; \eta) Z_{j}(\eta ; \zeta)\right| d \eta
$$

is convergent. Hence

$$
\begin{aligned}
\int_{\mathbb{R}^{N} \times[\tau, t]} Z_{1}(z ; \eta) \Phi(\eta ; \zeta) d \eta & =\sum_{j=1}^{\infty} \int_{\mathbb{R}^{N} \times[\tau, t]} Z_{1}(z ; \eta) Z_{j}(\eta ; \zeta) d \eta \\
& =\sum_{j=1}^{\infty} Z_{j+1}(z ; \zeta)=\Phi(z ; \zeta)-Z_{1}(z ; \zeta) .
\end{aligned}
$$

This ends the proof.

We shall need the following regularity properties of $\Phi$.

Proposition 2.3. Let $T>0$. We have

$$
\begin{aligned}
& \left|\Phi(x, t ; \xi, \tau)-\Phi\left(x^{\prime}, t ; \xi, \tau\right)\right| \\
& \leq \mathbf{c}(T) d\left(x, x^{\prime}\right)^{\frac{\alpha}{2}}(t-\tau)^{\frac{\alpha}{4}-1}\left(\mathbf{E}\left(\xi^{-1} \circ x, \mathbf{c}(t-\tau)\right)+\mathbf{E}\left(\xi^{-1} \circ x^{\prime}, \mathbf{c}(t-\tau)\right)\right),
\end{aligned}
$$

for every $x, x^{\prime}, \xi \in \mathbb{R}^{N}, 0<t-\tau \leq T$. Moreover, $\Phi(\cdot ; \zeta)$ and $\Phi(z ; \cdot)$ are continuous functions in their domains of definition.

We first prove the lemma below.

Lemma 2.4. For every $x, x^{\prime}, \xi \in \mathbb{R}^{N}$ and $t>\tau$, we have

$$
\begin{aligned}
& \left|Z_{1}(x, t ; \xi, \tau)-Z_{1}\left(x^{\prime}, t ; \xi, \tau\right)\right| \\
& \quad \leq \mathbf{c} d\left(x, x^{\prime}\right)^{\frac{\alpha}{2}}(t-\tau)^{\frac{\alpha}{4}-1}\left(\mathbf{E}\left(\xi^{-1} \circ x, \mathbf{c}(t-\tau)\right)+\mathbf{E}\left(\xi^{-1} \circ x^{\prime}, \mathbf{c}(t-\tau)\right)\right) .
\end{aligned}
$$


Proof. If $d\left(x, x^{\prime}\right) \geq \sqrt{t-\tau}$, it is sufficient to use (2.7). We thus suppose that $d\left(x, x^{\prime}\right)<\sqrt{t-\tau}$. From (2.1), (2.3) and (2.6), it follows that $(\zeta=(\xi, \tau))$

$$
\begin{aligned}
& \left|Z_{1}(x, t ; \zeta)-Z_{1}\left(x^{\prime}, t ; \zeta\right)\right|=\mid \sum_{i, j=1}^{m}\left(a_{i, j}(x, t)-a_{i, j}\left(x^{\prime}, t\right)\right) X_{i} X_{j} \Gamma_{\zeta}\left(\xi^{-1} \circ x^{\prime}, t-\tau\right) \\
& +\sum_{i, j=1}^{m}\left(a_{i, j}(x, t)-a_{i, j}(\zeta)\right)\left(X_{i} X_{j} \Gamma_{\zeta}\left(\xi^{-1} \circ x, t-\tau\right)-X_{i} X_{j} \Gamma_{\zeta}\left(\xi^{-1} \circ x^{\prime}, t-\tau\right)\right) \mid \\
& \leq \mathbf{c} d\left(x, x^{\prime}\right)^{\alpha}(t-\tau)^{-1} \mathbf{E}\left(\xi^{-1} \circ x^{\prime}, \mathbf{c}(t-\tau)\right)+\mathbf{c}\left(d(x, \xi)^{\alpha}+(t-\tau)^{\frac{\alpha}{2}}\right) \\
& \times \sum_{i, j=1}^{m}\left|X_{i} X_{j} \Gamma_{\zeta}\left(\xi^{-1} \circ x, t-\tau\right)-X_{i} X_{j} \Gamma_{\zeta}\left(\xi^{-1} \circ x^{\prime}, t-\tau\right)\right| .
\end{aligned}
$$

The summation in the far right-hand side can be estimated (via the mean value theorem on $X$-subunit paths; see, e.g., [2, Lemma 7.6]) by

$$
\mathbf{c} d\left(x, x^{\prime}\right) \sup _{d(y, x) \leq \mathbf{c} d\left(x, x^{\prime}\right)} \sum_{i, j, k=1}^{m}\left|X_{k} X_{i} X_{j} \Gamma_{\zeta}\left(\xi^{-1} \circ y, t-\tau\right)\right|
$$

Moreover, recalling (2.3) and using the fact that $d\left(x, x^{\prime}\right)<\sqrt{t-\tau}$, the supremum is lower than $\mathbf{c}(t-\tau)^{-3 / 2} \mathbf{E}\left(\xi^{-1} \circ x, \mathbf{c}(t-\tau)\right)$. Using again that $d\left(x, x^{\prime}\right)<\sqrt{t-\tau}$, we finally get

$$
\begin{aligned}
\left|Z_{1}(x, t ; \zeta)-Z_{1}\left(x^{\prime}, t ; \zeta\right)\right| \leq & \mathbf{c} d\left(x, x^{\prime}\right)^{\frac{\alpha}{2}}(t-\tau)^{\frac{\alpha}{4}-1}\left(\mathbf{E}\left(\xi^{-1} \circ x^{\prime}, \mathbf{c}(t-\tau)\right)\right. \\
& \left.+\left(1+\frac{d\left(\xi^{-1} \circ x\right)^{\alpha}}{(t-\tau)^{\frac{\alpha}{2}}}\right) \mathbf{E}\left(\xi^{-1} \circ x, \mathbf{c}(t-\tau)\right)\right),
\end{aligned}
$$

which gives the desired inequality, recalling (2.8).

Proof of Proposition 2.3. From (2.9) and Lemma 2.4 (and using (2.2) and (1.6)), it follows that

$$
\begin{aligned}
&\left|Z_{j+1}(x, t ; \xi, \tau)-Z_{j+1}\left(x^{\prime}, t ; \xi, \tau\right)\right| \\
& \leq \int_{\mathbb{R}^{N} \times[\tau, t]}\left|Z_{j}(y, s ; \xi, \tau)\right|\left|Z_{1}(x, t ; y, s)-Z_{1}\left(x^{\prime}, t ; y, s\right)\right| d y d s \\
& \leq \mathbf{c} b_{j}(\alpha) \mathbf{c}_{1}^{j} d\left(x, x^{\prime}\right)^{\frac{\alpha}{2}} \int_{\tau}^{t}(t-s)^{-1+\frac{\alpha}{4}}(s-\tau)^{-1+\frac{j \alpha}{2}} \int_{\mathbb{R}^{N}} \Gamma_{0}\left(\xi^{-1} \circ y, \mathbf{c}_{2}(s-\tau)\right) \\
& \times\left(\mathbf{E}\left(y^{-1} \circ x, \mathbf{c}(t-s)\right)+\mathbf{E}\left(y^{-1} \circ x^{\prime}, \mathbf{c}(t-s)\right)\right) d y d s \\
& \leq \mathbf{c} b_{j}\left(\frac{\alpha}{2}\right) \mathbf{c}_{1}^{j} d\left(x, x^{\prime}\right)^{\frac{\alpha}{2}} \int_{\tau}^{t}(t-s)^{-1+\frac{\alpha}{4}}(s-\tau)^{-1+\frac{j \alpha}{2}} \int_{\mathbb{R}^{N}} \Gamma_{0}\left(\xi^{-1} \circ y, \mathbf{c}_{3}(s-\tau)\right) \\
& \times\left(\Gamma_{0}\left(y^{-1} \circ x, \mathbf{c}_{3}(t-s)\right)+\Gamma_{0}\left(y^{-1} \circ x^{\prime}, \mathbf{c}_{3}(t-s)\right) d y d s\right. \\
&= \mathbf{c} d\left(x, x^{\prime}\right)^{\frac{\alpha}{2}}(t-\tau)^{-1+\frac{\alpha}{4}+\frac{j \alpha}{2}} b_{j}\left(\frac{\alpha}{2}\right) \mathbf{c}_{1}^{j} \int_{0}^{1}(1-r)^{-1+\frac{\alpha}{4}} r^{-1+\frac{j \alpha}{2}} d r \\
& \times\left(\Gamma_{0}\left(\xi^{-1} \circ x, \mathbf{c}_{3}(t-\tau)\right)+\Gamma_{0}\left(\xi^{-1} \circ x^{\prime}, \mathbf{c}_{3}(t-\tau)\right)\right) \\
& \leq \mathbf{c} d\left(x, x^{\prime}\right)^{\frac{\alpha}{2}}(t-\tau)^{-1+\frac{\alpha}{4}+\frac{j \alpha}{2}} b_{j+1}\left(\frac{\alpha}{2}\right) \mathbf{c}_{1}^{j} \\
& \times\left(\mathbf{E}\left(\xi^{-1} \circ x, \mathbf{c}(t-\tau)\right)+\mathbf{E}\left(\xi^{-1} \circ x^{\prime}, \mathbf{c}(t-\tau)\right)\right) .
\end{aligned}
$$


Therefore, using Lemma 2.4 again, we obtain

$$
\begin{aligned}
& \left|\Phi(x, t ; \xi, \tau)-\Phi\left(x^{\prime}, t ; \xi, \tau\right)\right| \leq\left|Z_{1}(x, t ; \xi, \tau)-Z_{1}\left(x^{\prime}, t ; \xi, \tau\right)\right| \\
& \quad+\sum_{j=1}^{\infty}\left|Z_{j+1}(x, t ; \xi, \tau)-Z_{j+1}\left(x^{\prime}, t ; \xi, \tau\right)\right| \\
& \leq \mathbf{c}\left(1+\sum_{j=1}^{\infty} b_{j+1}\left(\frac{\alpha}{2}\right)\left(\mathbf{c}_{1}(t-\tau)^{\frac{\alpha}{2}}\right)^{j}\right) \\
& \quad \times d\left(x, x^{\prime}\right)^{\frac{\alpha}{2}}(t-\tau)^{\frac{\alpha}{4}-1}\left(\mathbf{E}\left(\xi^{-1} \circ x, \mathbf{c}(t-\tau)\right)+\mathbf{E}\left(\xi^{-1} \circ x^{\prime}, \mathbf{c}(t-\tau)\right)\right),
\end{aligned}
$$

which proves (2.11).

We now turn to the proof of the last statement of Proposition 2.3. Since (2.11) holds, in order to prove the continuity of $\Phi(\cdot ; \zeta)$, we only have to see that $\Phi(x, \cdot ; \zeta)$ is continuous. This will follow from the continuity of $Z_{j}(x, \cdot ; \zeta), j \in \mathbb{N}$. For $j=1$, such continuity follows immediately from the definition of $Z_{1}$. We now fix $j \in \mathbb{N}$ and prove that $Z_{j+1}(x, \cdot ; \zeta)$ is continuous at $t_{0}>\tau$. We have

$$
\begin{aligned}
& \left|Z_{j+1}(x, t ; \zeta)-Z_{j+1}\left(x, t_{0} ; \zeta\right)\right| \\
& \leq \int_{\tau}^{t_{0}-\delta} \int_{\mathbb{R}^{N}}\left|Z_{j}(y, s ; \zeta)\right|\left|Z_{1}\left(x, t_{0} ; y, s\right)-Z_{1}(x, t ; y, s)\right| d y d s \\
& \quad+\int_{t_{0}-\delta}^{t} \int_{\mathbb{R}^{N}}\left|Z_{j}(y, s ; \zeta) Z_{1}(x, t ; y, s)\right| d y d s \\
& \quad+\int_{t_{0}-\delta}^{t_{0}} \int_{\mathbb{R}^{N}}\left|Z_{j}(y, s ; \zeta) Z_{1}\left(x, t_{0} ; y, s\right)\right| d y d s .
\end{aligned}
$$

By using (2.7) and (2.9), it is easy to see that the last two integrals in the righthand side are small if $\left|t-t_{0}\right|<\delta$ and $\delta$ is small enough. On the other hand, from (2.1), (2.3) and (2.6) it follows that, for $\left|t-t_{0}\right|<\delta / 2$ and for every $s \in\left(\tau, t_{0}-\delta\right)$, we have

$$
\begin{aligned}
& \left|Z_{1}\left(x, t_{0} ; y, s\right)-Z_{1}(x, t ; y, s)\right| \\
& \leq \sum_{i, j=1}^{m}\left(\left|a_{i, j}\left(x, t_{0}\right)-a_{i, j}(x, t)\right|\right. \\
& \quad \times\left|X_{i} X_{j} \Gamma_{(y, s)}\left(y^{-1} \circ x, t_{0}-s\right)\right|+\left|a_{i, j}(x, t)-a_{i, j}(y, s)\right| \\
& \left.\quad \times\left|X_{i} X_{j} \Gamma_{(y, s)}\left(y^{-1} \circ x, t_{0}-s\right)-X_{i} X_{j} \Gamma_{(y, s)}\left(y^{-1} \circ x, t-s\right)\right|\right) \\
& \leq \mathbf{c}\left(\left|t-t_{0}\right|^{\frac{\alpha}{2}}\left(t_{0}-s\right)^{-1} \mathbf{E}\left(y^{-1} \circ x, \mathbf{c}\left(t_{0}-s\right)\right)\right. \\
& \left.\quad \quad+\left|t-t_{0}\right| \sum_{i, j=1}^{m} \sup _{h \geq \delta / 2}\left|\partial_{t} X_{i} X_{j} \Gamma_{(y, s)}\left(y^{-1} \circ x, h\right)\right|\right) \\
& \leq \mathbf{c}(\delta)\left|t-t_{0}\right|^{\frac{\alpha}{2}} .
\end{aligned}
$$

As a consequence, using also (1.5) and (2.9), for $\left|t-t_{0}\right|<\delta / 2$ we get

$$
\begin{aligned}
\int_{\tau}^{t_{0}-} & \delta \\
& \int_{\mathbb{R}^{N}}\left|Z_{j}(y, s ; \zeta)\right|\left|Z_{1}\left(x, t_{0} ; y, s\right)-Z_{1}(x, t ; y, s)\right| d y d s \\
\leq & \mathbf{c}(\delta, j)\left|t-t_{0}\right|^{\frac{\alpha}{2}} \int_{\tau}^{t_{0}}(s-\tau)^{-1+\frac{j \alpha}{2}}\left(\int_{\mathbb{R}^{N}} \Gamma_{0}\left(\xi^{-1} \circ y, \mathbf{c}(s-\tau)\right) d y\right) d s \\
= & \mathbf{c}(\delta, j)\left|t-t_{0}\right|^{\frac{\alpha}{2}} \int_{\tau}^{t_{0}}(s-\tau)^{-1+\frac{j \alpha}{2}} d s \leq \mathbf{c}\left(\delta, j, t_{0}, \tau\right)\left|t-t_{0}\right|^{\frac{\alpha}{2}}
\end{aligned}
$$

In this way the continuity at $t_{0}$ of $Z_{j+1}(x, \cdot ; \zeta)$ is proved. 
It remains only to prove that $\Phi(z ; \cdot)$ is continuous. To this end, it is sufficient to show the continuity of $Z_{j}(z ; \cdot)$ for every $j \in \mathbb{N}$. For $j=1$, it is easy to see that $Z_{1}(z ; \cdot)$ is continuous by using (2.1), (2.5) and (2.6). One can then prove the continuity of $Z_{j}(z ; \cdot)$ by induction, showing that $Z_{j+1}(z ; \cdot)$ is a uniform limit, as $\sigma \rightarrow 0^{+}$, of the continuous functions $\zeta=(\xi, \tau) \mapsto \int_{\mathbb{R}^{N} \times[\tau+\sigma, t]} Z_{1}(z ; \eta) Z_{j}(\eta ; \zeta) d \eta$, on the compact subsets of $\mathbb{R}^{N} \times(-\infty, t)$ (by using the estimate [2.9); see also the proof of Proposition 2.1).

We now set, for every $z=(x, t), \zeta=(\xi, \tau) \in \mathbb{R}^{N+1}, t>\tau$,

$$
J(z ; \zeta)=\int_{\mathbb{R}^{N} \times[\tau, t]} \Gamma_{\eta}(z ; \eta) \Phi(\eta ; \zeta) d \eta
$$

and we define

$$
\Gamma(z ; \zeta)=J(z ; \zeta)+\Gamma_{\zeta}(z ; \zeta)
$$

We also agree to extend $\Gamma(z ; \zeta)$ to be zero for $t \leq \tau$. We remark that the integral in (2.12) is convergent since, by means of (1.6), (2.2) and (2.10), we have (for $0<t-\tau \leq T)$

$$
\begin{aligned}
& \int_{\mathbb{R}^{N} \times[\tau, t]}\left|\Gamma_{\eta}(z ; \eta) \Phi(\eta ; \zeta)\right| d \eta \\
& \quad \leq \mathbf{c}(T) \int_{\tau}^{t}(s-\tau)^{-1+\frac{\alpha}{2}} \int_{\mathbb{R}^{N}} \Gamma_{0}\left(y^{-1} \circ x, \mathbf{c}_{1}(t-s)\right) \Gamma_{0}\left(\xi^{-1} \circ y, \mathbf{c}_{1}(s-\tau)\right) d y d s \\
& \quad=\mathbf{c}(T)(t-\tau)^{\frac{\alpha}{2}} \Gamma_{0}\left(\xi^{-1} \circ x, \mathbf{c}_{1}(t-\tau)\right) .
\end{aligned}
$$

This also proves that $J$ satisfies the following estimate, for $0<t-\tau \leq T$ :

$$
|J(z ; \zeta)| \leq \mathbf{c}(T)(t-\tau)^{\frac{\alpha}{2}} \mathbf{E}\left(\xi^{-1} \circ x, \mathbf{c}(t-\tau)\right) .
$$

Proposition 2.5. The function $\Gamma$ is continuous away from the diagonal of $\mathbb{R}^{N+1} \times$ $\mathbb{R}^{N+1}$. Moreover, $\Gamma$ satisfies the following estimate:

$$
|\Gamma(z ; \zeta)| \leq \mathbf{c}(T) \mathbf{E}\left(\xi^{-1} \circ x, \mathbf{c}(t-\tau)\right),
$$

for every $z=(x, t), \zeta=(\xi, \tau) \in \mathbb{R}^{N+1}, 0<t-\tau \leq T$.

Proof. (2.16) immediately follows from (2.2) and (2.15), recalling the definition (2.13) of $\Gamma$. Moreover, (2.16) gives the continuity of $\Gamma(z ; \zeta)$ in the set $\{t=\tau, x \neq \xi\}$. Therefore, we only have to prove that $\Gamma$ is continuous in the set $\{t>\tau\}$. Since (2.5) holds, the function $(z ; \zeta) \mapsto \Gamma_{\zeta}(z ; \zeta)$ is continuous away from the diagonal $\{z=\zeta\}$. Hence, it is sufficient to see that $J$ is continuous in $\{t>\tau\}$. This can be done by showing that the functions

$$
J_{\sigma}(z ; \zeta)=\int_{\mathbb{R}^{N} \times[\tau+\sigma, t-\sigma]} \Gamma_{\eta}(z ; \eta) \Phi(\eta ; \zeta) d \eta
$$

are continuous and converge uniformly to $J$, as $\sigma \rightarrow 0^{+}$, on the compact subsets of $\{t>\tau\}$. The continuity of $J_{\sigma}$ follows from the continuity of $\Gamma_{\eta}$ and of $\Phi(\eta ; \cdot)$ (see Proposition [2.3), by dominated convergence, using the estimates (2.2) and (2.10). 
On the other hand, for every $K \Subset\{t>\tau\}$,

$$
\begin{aligned}
& \sup _{K}\left|J_{\sigma}-J\right| \leq \sup _{(z, \zeta) \in K}\left(\int_{\tau}^{\tau+\sigma}+\int_{t-\sigma}^{t}\right) \int_{\mathbb{R}^{N}}\left|\Gamma_{\eta}(z ; \eta) \Phi(\eta ; \zeta)\right| d \eta \\
& \leq \mathbf{c}(K) \sup _{(z, \zeta) \in K}\left(\int_{\tau}^{\tau+\sigma}+\int_{t-\sigma}^{t}\right)(s-\tau)^{-1+\frac{\alpha}{2}} \int_{\mathbb{R}^{N}} \Gamma_{0}\left(y^{-1} \circ x, \mathbf{c}_{1}(t-s)\right) \\
& \quad \times \Gamma_{0}\left(\xi^{-1} \circ y, \mathbf{c}_{1}(s-\tau)\right) d y d s \\
& =\mathbf{c}(K) \sup _{(z, \zeta) \in K} \Gamma_{0}\left(\xi^{-1} \circ x, \mathbf{c}_{1}(t-\tau)\right)\left(\int_{\tau}^{\tau+\sigma}+\int_{t-\sigma}^{t}\right)(s-\tau)^{-1+\frac{\alpha}{2}} d s \\
& \leq \mathbf{c}(K) \sigma^{\alpha / 2} \longrightarrow 0 .
\end{aligned}
$$

Here we used the estimates (1.6), (2.2) and (2.10).

We now want to show that $\Gamma$ is the fundamental solution for $\mathcal{H}$. Our first goal is to prove that $\mathcal{H}(\Gamma(\cdot ; \zeta))=0$ in $\mathbb{R}^{N+1} \backslash\{\zeta\}$. We start by giving the definition of a regularity class of functions that will be useful in the sequel.

Definition 2.6. Let $\Omega \subseteq \mathbb{R}^{N+1}$ be an open set. We denote by $\mathfrak{C}^{2}(\Omega)$ the class of functions $u(x, t)$ defined on $\Omega$ which are continuous in $\Omega$ w.r.t. the pair $(x, t)$ and such that $u(\cdot, t)$ has continuous Lie derivatives up to second order along the vector fields $X_{1}, \ldots, X_{m}$ (w.r.t. $x$, for every fixed $t$ ) and $u(x, \cdot)$ has continuous derivative (w.r.t. $t$, for every fixed $x$ ), in their respective domains of definition.

We recall that the Lie derivative along a vector field $X \in \mathfrak{g}$ of a function $v(x)$ at a point $x_{0} \in \mathbb{R}^{N}$ is defined to be

$$
X v\left(x_{0}\right)=\left.\frac{d}{d h}\right|_{h=0} v\left(x_{0} \circ \mu_{X}(h)\right)
$$

(if such a derivative exists), where $\mu_{X}$ is the solution to the ODE $\dot{\mu}(h)=X(\mu(h))$, $\mu(0)=0$. The class $\mathfrak{C}^{2}(\Omega)$ is larger than the $\Gamma^{2}(\Omega)$ introduced in Section 1 nevertheless we are able to prove a weak maximum principle for solutions to $\mathcal{H} u \geq 0$ in the class $\mathfrak{C}^{2}(\Omega)$ (see Theorem 4.1).

Theorem 2.7. For every fixed $\zeta \in \mathbb{R}^{N+1}$, we have

$$
\Gamma(\cdot ; \zeta) \in \mathfrak{C}^{2}\left(\mathbb{R}^{N+1} \backslash\{\zeta\}\right), \quad \mathcal{H}(\Gamma(\cdot ; \zeta))=0 \text { in } \mathbb{R}^{N+1} \backslash\{\zeta\} .
$$

Moreover, the following estimates hold for $0<t-\tau \leq T$ :

$$
\begin{gathered}
\left|X_{j}(\Gamma(\cdot ; \zeta))(z)\right| \leq \mathbf{c}(T)(t-\tau)^{-1 / 2} \mathbf{E}\left(\xi^{-1} \circ x, \mathbf{c}(t-\tau)\right) ; \\
\left|X_{i} X_{j}(\Gamma(\cdot ; \zeta))(z)\right|,\left|\partial_{t}(\Gamma(\cdot ; \zeta))(z)\right| \leq \mathbf{c}(T)(t-\tau)^{-1} \mathbf{E}\left(\xi^{-1} \circ x, \mathbf{c}(t-\tau)\right) .
\end{gathered}
$$

In the proof of Theorem 2.7 we shall use the following simple result.

Lemma 2.8. Let $X \in \mathfrak{g}$ and let $\left\{u_{j}\right\}_{j}$ be a sequence of continuous functions, defined on an open set $A \subseteq \mathbb{R}^{N}$, with continuous Lie derivative along $X$. Suppose that $u_{j}$ converges pointwise in $A$ to some function $u$ and that $X u_{j}$ converges to some function $w$ uniformly on the compact subsets of $A$. Then there exists the Lie derivative of $u$ along $X, X u(x)=w(x)$, for every $x \in A$.

Proof. Given a non-negative function $\Psi \in C_{0}^{\infty}\left(B_{d}(0,1)\right)$ such that $\Psi\left(x^{-1}\right)=\Psi(x)$, $\int \Psi=1$, we define the $\mathbb{G}$-regularization

$$
u_{j}^{\varepsilon}(x)=\int \Psi_{\varepsilon}\left(y \circ x^{-1}\right) u_{j}(y) d y
$$


(where $\Psi_{\varepsilon}=\varepsilon^{-Q} \Psi\left(\delta_{\varepsilon^{-1}}(\cdot)\right)$ ) in such a way that $X\left(u_{j}^{\varepsilon}\right)=\left(X u_{j}\right)^{\varepsilon}$ (see, e.g., [42]). We have

$$
\begin{aligned}
& \frac{1}{h}\left(u_{j}^{\varepsilon}\left(x \circ \mu_{X}(h)\right)-u_{j}^{\varepsilon}(x)\right)=\frac{1}{h} \int_{0}^{h} \frac{d}{d s}\left(u_{j}^{\varepsilon}\left(x \circ \mu_{X}(s)\right)\right) d s \\
& =\frac{1}{h} \int_{0}^{h} X u_{j}^{\varepsilon}\left(x \circ \mu_{X}(s)\right) d s=\frac{1}{h} \int_{0}^{h}\left(X u_{j}\right)^{\varepsilon}\left(x \circ \mu_{X}(s)\right) d s .
\end{aligned}
$$

Letting first $\varepsilon$ go to zero, then $j$ go to infinity and finally $h$ go to zero, one proves that there exists $X u(x)=w(x)$.

Remark 2.9. Let $X \in \mathfrak{g}$. If $u$ is a continuous function on an open set $A \subseteq \mathbb{R}^{N}$ and the Lie derivative $X u$ exists (in the point-wise sense (2.17)) and is continuous in $A$, then $X u$ is also a derivative in the weak sense of distributions.

Proof. We first observe that, if $u, X u$ are continuous, then $X u(x)$ is a uniform limit of $\left(u\left(x \circ \mu_{X}(h)\right)-u(x)\right) / h$ as $h \rightarrow 0$, on the compact subsets of $A$. This is evident from the proof of Lemma 2.8. Let $\varphi \in C_{0}^{\infty}(A)$, and let us set for brevity $\tilde{\varphi}(y)=\left.\frac{d}{d h}\right|_{h=0} \varphi\left(y \circ\left(\mu_{X}(h)\right)^{-1}\right)$. For every test function $\psi \in C_{0}^{\infty}(A)$ we have

$$
\begin{aligned}
\int \psi X^{*} \varphi & =\int \varphi X \psi \stackrel{h \rightarrow 0}{\longleftarrow} \int \frac{1}{h} \varphi(x)\left(\psi\left(x \circ \mu_{X}(h)\right)-\psi(x)\right) d x \\
& =\int \frac{1}{h} \psi(y)\left(\varphi\left(y \circ\left(\mu_{X}(h)\right)^{-1}\right)-\varphi(y)\right) d y \stackrel{h \rightarrow 0}{\longrightarrow} \int \psi \widetilde{\varphi}
\end{aligned}
$$

Hence $X^{*} \varphi=\widetilde{\varphi}$, and we have

$$
\begin{aligned}
\int \varphi X u & \stackrel{h \rightarrow 0}{\longleftarrow} \int \frac{1}{h} \varphi(x)\left(u\left(x \circ \mu_{X}(h)\right)-u(x)\right) d x \\
& =\int \frac{1}{h} u(y)\left(\varphi\left(y \circ\left(\mu_{X}(h)\right)^{-1}\right)-\varphi(y)\right) d y \stackrel{h \rightarrow 0}{\longrightarrow} \int u \widetilde{\varphi}=\int u X^{*} \varphi .
\end{aligned}
$$

This ends the proof.

The main step in the proof of Theorem 2.7 is the following lemma.

Lemma 2.10. For every fixed $\zeta=(\xi, \tau) \in \mathbb{R}^{N+1}$, the function $J(\cdot ; \zeta)$ is in $\mathfrak{C}^{2}\left(\left\{z=(x, t) \in \mathbb{R}^{N+1}: t>\tau\right\}\right)$, and we have (we use the notation $\eta=(y, s)$ )

$$
\begin{gathered}
X_{j}(J(\cdot ; \zeta))(z)=\int_{\mathbb{R}^{N} \times[\tau, t]} X_{j} \Gamma_{\eta}\left(y^{-1} \circ x, t-s\right) \Phi(\eta ; \zeta) d \eta \\
X_{i} X_{j}(J(\cdot ; \zeta))(z)=\lim _{\varepsilon \rightarrow 0^{+}} \int_{\mathbb{R}^{N} \times[\tau, t-\varepsilon]} X_{i} X_{j} \Gamma_{\eta}\left(y^{-1} \circ x, t-s\right) \Phi(\eta ; \zeta) d \eta \\
\partial_{t}(J(\cdot ; \zeta))(z)=\Phi(z ; \zeta)+\lim _{\varepsilon \rightarrow 0^{+}} \int_{\mathbb{R}^{N} \times[\tau, t-\varepsilon]} \partial_{t} \Gamma_{\eta}\left(y^{-1} \circ x, t-s\right) \Phi(\eta ; \zeta) d \eta
\end{gathered}
$$

Moreover, the following estimates hold for $0<t-\tau \leq T$ :

$$
\begin{aligned}
\left|X_{j}(J(\cdot ; \zeta))(z)\right| & \leq \mathbf{c}(T)(t-\tau)^{(\alpha-1) / 2} \mathbf{E}\left(\xi^{-1} \circ x, \mathbf{c}(t-\tau)\right) \\
\left|X_{i} X_{j}(J(\cdot ; \zeta))(z)\right|, & \left|\partial_{t}(J(\cdot ; \zeta))(z)\right| \\
\leq & \mathbf{c}(T)(t-\tau)^{(\alpha-2) / 2} \mathbf{E}\left(\xi^{-1} \circ x, \mathbf{c}(t-\tau)\right) .
\end{aligned}
$$


Proof. The continuity of $J(\cdot ; \zeta)$ has been proved in Proposition 2.5. In order to prove that the Lie derivatives in (2.22) and (2.23) exist, we shall use Lemma (2.8). Let us set

$$
J_{\varepsilon}(z ; \zeta)=\int_{\mathbb{R}^{N} \times[\tau, t-\varepsilon]} \Gamma_{\eta}(z ; \eta) \Phi(\eta ; \zeta) d \eta,
$$

so that $J_{\varepsilon}$ converges pointwise to $J$, as $\varepsilon \rightarrow 0^{+}$. It is not difficult to see that $J_{\varepsilon}(\cdot, t ; \zeta)$ has continuous Lie derivatives up to second order along the vector fields $X_{1}, \ldots, X_{m}$, obtained deriving (2.27) under the integral sign. In order to prove $(2.22)$ it is then sufficient to show that

$$
\sup _{x \in \mathbb{R}^{N}} \int_{\mathbb{R}^{N} \times[t-\varepsilon, t]}\left|X_{j} \Gamma_{\eta}\left(y^{-1} \circ x, t-s\right) \Phi(\eta ; \zeta)\right| d \eta \longrightarrow 0, \quad \text { as } \varepsilon \rightarrow 0^{+},
$$

which is an easy consequence of the estimates (2.3) and (2.10).

In order to prove (2.23), we now only have to show that the limit in (2.23) exists, uniformly in $x \in \mathbb{R}^{N}$. To this end, let us consider the integral

$$
I=\int_{\mathbb{R}^{N}} X_{i} X_{j} \Gamma_{\eta}\left(y^{-1} \circ x, t-s\right) \Phi(\eta ; \zeta) d y, \quad \tau<s<t .
$$

Using (2.2), (2.3), (2.10) and (1.6), it is easy to see, as in (2.14), that

$$
|I| \leq \mathbf{c}(T)(t-s)^{-1}(s-\tau)^{-1+\frac{\alpha}{2}} \mathbf{E}\left(\xi^{-1} \circ x, \mathbf{c}(t-\tau)\right) .
$$

Moreover, for every fixed $y_{0} \in \mathbb{R}^{N}$, we have $I=I_{1}+I_{2}+I_{3}$, where

$$
\begin{aligned}
I_{1} & =\int_{\mathbb{R}^{N}} X_{i} X_{j} \Gamma_{\eta}\left(y^{-1} \circ x, t-s\right)\left(\Phi(y, s ; \zeta)-\Phi\left(y_{0}, s ; \zeta\right)\right) d y, \\
I_{2} & =\Phi\left(y_{0}, s ; \zeta\right) \int_{\mathbb{R}^{N}} X_{i} X_{j}\left(\Gamma_{(y, s)}-\Gamma_{\left(y_{0}, s\right)}\right)\left(y^{-1} \circ x, t-s\right) d y, \\
I_{3} & =\Phi\left(y_{0}, s ; \zeta\right) \int_{\mathbb{R}^{N}} X_{i} X_{j} \Gamma_{\left(y_{0}, s\right)}\left(y^{-1} \circ x, t-s\right) d y .
\end{aligned}
$$

Since $\int_{\mathbb{R}^{N}} \Gamma_{\left(y_{0}, s\right)}\left(y^{-1} \circ x, t-s\right) d y=1$ by (1.5)), deriving under the integral sign (recalling the estimate $(2.3)$ ), we obtain $I_{3} \equiv 0$. We now choose $y_{0}=x$ and we estimate $I_{1}, I_{2}$. Making use of (1.5), (1.6), (2.2), (2.3), (2.8) and (2.11), we get

$$
\begin{aligned}
\left|I_{1}\right| \leq & \mathbf{c}(T)(t-s)^{-1}(s-\tau)^{-1+\frac{\alpha}{4}} \int_{\mathbb{R}^{N}} d(x, y)^{\frac{\alpha}{2}} \mathbf{E}\left(y^{-1} \circ x, \mathbf{c}(t-s)\right) \\
& \times\left(\mathbf{E}\left(\xi^{-1} \circ y, \mathbf{c}(s-\tau)\right)+\mathbf{E}\left(\xi^{-1} \circ x, \mathbf{c}(s-\tau)\right)\right) d y \\
\leq & \mathbf{c}(T)((t-s)(s-\tau))^{-1+\frac{\alpha}{4}}\left(\mathbf{E}\left(\xi^{-1} \circ x, \mathbf{c}(s-\tau)\right) \int_{\mathbb{R}^{N}} \Gamma_{0}\left(y^{-1} \circ x, \mathbf{c}(t-s)\right) d y\right. \\
& \left.+\int_{\mathbb{R}^{N}} \Gamma_{0}\left(\xi^{-1} \circ y, \mathbf{c}_{1}(s-\tau)\right) \Gamma_{0}\left(y^{-1} \circ x, \mathbf{c}_{1}(t-s)\right) d y\right) \\
\leq & \mathbf{c}(T)((t-s)(s-\tau))^{-1+\frac{\alpha}{4}}\left(\mathbf{E}\left(\xi^{-1} \circ x, \mathbf{c}(t-\tau)\right)+\mathbf{E}\left(\xi^{-1} \circ x, \mathbf{c}(s-\tau)\right)\right) .
\end{aligned}
$$

Using (1.5), (2.2), (2.5), (2.8) and (2.10), we obtain

$$
\begin{aligned}
\left|I_{2}\right| & \leq \mathbf{c}(T)(s-\tau)^{-1+\frac{\alpha}{2}} \mathbf{E}\left(\xi^{-1} \circ x, \mathbf{c}(s-\tau)\right) \\
& \times \int_{\mathbb{R}^{N}} d(x, y)^{\frac{\alpha}{r}}(t-s)^{-1} \mathbf{E}\left(y^{-1} \circ x, \mathbf{c}(t-s)\right) d y \\
& \leq \mathbf{c}(T)(t-s)^{-1+\frac{\alpha}{2 r}}(s-\tau)^{-1+\frac{\alpha}{2}} \mathbf{E}\left(\xi^{-1} \circ x, \mathbf{c}(s-\tau)\right) .
\end{aligned}
$$


Collecting the above estimates, it is now immediate to recognize that the limit in (2.23) exists, and it is uniform in $x \in \mathbb{R}^{N}$.

In order to conclude the proof of the first statement of Lemma 2.10, it remains only to prove that $J(x, \cdot ; \zeta)$ has continuous derivative given by (2.24). To this end, it is sufficient to show that $J_{\varepsilon}(x, \cdot ; \zeta)$ has continuous derivative given by

$$
\begin{aligned}
\partial_{t} J_{\varepsilon}(x, t ; \zeta)= & \int_{\mathbb{R}^{N}} \Gamma_{(y, t-\varepsilon)}\left(y^{-1} \circ x, \varepsilon\right) \Phi(y, t-\varepsilon ; \zeta) d y \\
& +\int_{\mathbb{R}^{N} \times[\tau, t-\varepsilon]} \partial_{t} \Gamma_{(y, s)}\left(y^{-1} \circ x, t-s\right) \Phi(y, s ; \zeta) d y d s
\end{aligned}
$$

and that

$$
\begin{aligned}
& \sup _{t \in K}\left|\Phi(z ; \zeta)-\int_{\mathbb{R}^{N}} \Gamma_{(y, t-\varepsilon)}\left(y^{-1} \circ x, \varepsilon\right) \Phi(y, t-\varepsilon ; \zeta) d y\right| \longrightarrow 0, \\
& \sup _{t \in K} \int_{t-\varepsilon}^{t}\left|\int_{\mathbb{R}^{N}} \partial_{t} \Gamma_{(y, s)}\left(y^{-1} \circ x, t-s\right) \Phi(y, s ; \zeta) d y\right| d s \longrightarrow 0,
\end{aligned}
$$

as $\varepsilon \rightarrow 0^{+}$, for every $K \Subset(\tau, \infty)$. We have

$$
\begin{aligned}
& \frac{1}{h}\left(J_{\varepsilon}(x, t+h ; \zeta)-J_{\varepsilon}(x, t ; \zeta)\right) \\
& =\int_{t-\varepsilon}^{t-\varepsilon+h} \frac{1}{h} \int_{\mathbb{R}^{N}} \Gamma_{(y, s)}\left(y^{-1} \circ x, t+h-s\right) \Phi(y, s ; \zeta) d y d s \\
& \quad+\int_{\mathbb{R}^{N} \times[\tau, t-\varepsilon]} \frac{1}{h}\left(\Gamma_{\eta}\left(y^{-1} \circ x, t+h-s\right)-\Gamma_{\eta}\left(y^{-1} \circ x, t-s\right)\right) \Phi(\eta ; \zeta) d \eta .
\end{aligned}
$$

The second integral in (2.34) converges (as $h \rightarrow 0$ ) to the second integral in (2.31), by dominated convergence (recalling (2.3) and (2.10) $)$. The first integral in (2.34) is equal to

$$
\int_{0}^{1} \int_{\mathbb{R}^{N}} \Gamma_{(y, t-\varepsilon+r h)}\left(y^{-1} \circ x, \varepsilon+h-r h\right) \Phi(y, t-\varepsilon+r h ; \zeta) d y d r
$$

which converges to the first integral in (2.31) (as $h \rightarrow 0$ ) by dominated convergence, by means of (2.2), (2.5), (2.10) and Proposition 2.3. This proves (2.31). Using the properties just recalled, it is also easy to see that $\partial_{t} J_{\varepsilon}(x, \cdot ; \zeta)$ is continuous, again by dominated convergence. We now prove (2.32) and (2.33). Recalling (1.5), the supremum in (2.32) is lower than $S_{1}+S_{2}+S_{3}$, where

$$
\begin{aligned}
S_{1} & =\sup _{t \in K} \int_{\mathbb{R}^{N}}\left|\left(\Gamma_{(y, t-\varepsilon)}\left(y^{-1} \circ x, \varepsilon\right)-\Gamma_{(x, t-\varepsilon)}\left(y^{-1} \circ x, \varepsilon\right)\right) \Phi(y, t-\varepsilon ; \zeta)\right| d y, \\
S_{2} & =\sup _{t \in K} \int_{\mathbb{R}^{N}} \Gamma_{(x, t-\varepsilon)}\left(y^{-1} \circ x, \varepsilon\right)|\Phi(y, t-\varepsilon ; \zeta)-\Phi(x, t-\varepsilon ; \zeta)| d y, \\
S_{3} & =\sup _{t \in K}|\Phi(x, t-\varepsilon ; \zeta)-\Phi(x, t ; \zeta)| .
\end{aligned}
$$


From the continuity of $\Phi(x, \cdot ; \zeta)$ (see Proposition [2.3), we infer that $S_{3} \rightarrow 0$ as $\varepsilon \rightarrow 0^{+}$. Using (1.5), (2.2), (2.5) and (2.10), we obtain

$$
\begin{aligned}
S_{1} & \left.\leq \mathbf{c}(K, \tau)\left(\int_{d(x, y) \leq \delta} d(x, y)^{\frac{\alpha}{r}} \mathbf{E}\left(y^{-1} \circ x, \mathbf{c} \varepsilon\right)\right) d y+2 \int_{d(x, y)>\delta} \mathbf{E}\left(y^{-1} \circ x, \mathbf{c} \varepsilon\right) d y\right) \\
& \leq \mathbf{c}(K, \tau)\left(\delta^{\frac{\alpha}{r}} \int_{\mathbb{R}^{N}} \Gamma_{0}\left(y^{-1} \circ x, \mathbf{c} \varepsilon\right) d y+\int_{d\left(y^{\prime}\right)>\frac{\delta}{\sqrt{\varepsilon}}} \exp \left(-\mathbf{c} d\left(y^{\prime}\right)^{2}\right) d y^{\prime}\right) \\
& \leq \mathbf{c}(K, \tau)\left(\delta^{\frac{\alpha}{r}}+\int_{d\left(y^{\prime}\right)>\frac{\delta}{\sqrt{\varepsilon}}} \exp \left(-\mathbf{c} d\left(y^{\prime}\right)^{2}\right) d y^{\prime}\right) .
\end{aligned}
$$

Hence $S_{1} \rightarrow 0$, as $\varepsilon \rightarrow 0^{+}$. In a similar manner (exploiting (2.11) ), one can see that also $S_{2}$ vanishes as $\varepsilon$ goes to zero. This proves (2.32). The proof of (2.33) closely follows the lines of the proof of (2.23), and therefore it is omitted.

We finally turn to the proof of the second statement of Lemma 2.10 The estimate (2.25) can be obtained arguing as in (2.14) and using (2.3). Moreover, from (2.28), $(2.29)$ and (2.30), it follows that

$$
\begin{aligned}
& \left|X_{i} X_{j}(J(\cdot ; \zeta))(z)\right| \leq \mathbf{c}(T) \mathbf{E}\left(\xi^{-1} \circ x, \mathbf{c}(t-\tau)\right)\left(\int_{\tau}^{(t+\tau) / 2}(t-s)^{-1}(s-\tau)^{-1+\frac{\alpha}{2}} d s\right. \\
& \left.\quad+\int_{(t+\tau) / 2}^{t}\left(((t-s)(s-\tau))^{-1+\frac{\alpha}{4}}+(t-s)^{-1+\frac{\alpha}{2 r}}(s-\tau)^{-1+\frac{\alpha}{2}}\right) d s\right) \\
& \leq \mathbf{c}(T)(t-\tau)^{-1+\frac{\alpha}{2}} \mathbf{E}\left(\xi^{-1} \circ x, \mathbf{c}(t-\tau)\right) .
\end{aligned}
$$

The estimate of $\partial_{t} J(x, \cdot ; \zeta)$ is analogous (also recalling (2.10) ).

Proof of Theorem 2.7. By means of Lemma 2.10 (and recalling (2.3) and Proposition 2.5), we only have to prove that $\mathcal{H}(\Gamma(\cdot ; \zeta))(z)=0$ for $t>\tau$. Making use of (2.23) and (2.24), we obtain

$$
\begin{aligned}
& \mathcal{H}(\Gamma(\cdot ; \zeta))(z) \\
& \quad=\mathcal{H}\left(\Gamma_{\zeta}(\cdot ; \zeta)\right)(z)+\lim _{\varepsilon \rightarrow 0^{+}} \int_{\mathbb{R}^{N} \times[\tau, t-\varepsilon]} \mathcal{H}\left(\Gamma_{\eta}(\cdot ; \eta)\right)(z) \Phi(\eta ; \zeta) d \eta-\Phi(z ; \zeta) \\
& \quad=Z_{1}(z ; \zeta)+\int_{\mathbb{R}^{N} \times[\tau, t]} Z_{1}(z ; \eta) \Phi(\eta ; \zeta) d \eta-\Phi(z ; \zeta)=0,
\end{aligned}
$$

by means of (2.5) and Corollary 2.2. This completes the proof.

\section{The Cauchy problem for $\mathcal{H}$}

The aim of this section is to prove the following theorem.

Theorem 3.1. There exists a positive constant $\delta$ such that the following statement holds for every $\mu \geq 0$ and $T_{2}>T_{1}$ satisfying $\left(T_{2}-T_{1}\right) \mu<\delta$. Given a continuous function $f(x, t)$ on $\mathbb{R}^{N} \times\left[T_{1}, T_{2}\right]$, locally Hölder continuous in $x$, uniformly w.r.t. $t$, and given a continuous function $g(x)$ on $\mathbb{R}^{N}$, satisfying the growth condition

$$
|f(x, t)|,|g(x)| \leq M \exp \left(\mu d^{2}(x)\right)
$$

for some constant $M>0$, the function (here $x \in \mathbb{R}^{N}, t \in\left(T_{1}, T_{2}\right]$ )

$$
u(x, t)=\int_{\mathbb{R}^{N}} \Gamma\left(x, t ; \xi, T_{1}\right) g(\xi) d \xi+\int_{\mathbb{R}^{N} \times\left[T_{1}, t\right]} \Gamma(x, t ; \xi, \tau) f(\xi, \tau) d \xi d \tau
$$


belongs to the class $\mathfrak{C}^{2}\left(\mathbb{R}^{N} \times\left(T_{1}, T_{2}\right)\right) \cap C\left(\mathbb{R}^{N} \times\left[T_{1}, T_{2}\right]\right)$ and is a solution to the following Cauchy problem:

$$
\left\{\begin{array}{l}
\mathcal{H} u=-f \quad \text { in } \mathbb{R}^{N} \times\left(T_{1}, T_{2}\right), \\
u\left(\cdot, T_{1}\right)=g .
\end{array}\right.
$$

We split the proof of Theorem 3.1 into lemmas.

Lemma 3.2. The statement of Theorem 3.1 holds if $f \equiv 0$.

Proof. We only prove that $u(x, t) \rightarrow g\left(x_{0}\right)$, as $(x, t) \rightarrow\left(x_{0}, T_{1}\right)$, for every fixed $x_{0} \in \mathbb{R}^{N}$. The other properties of $u$ easily follow from Proposition 2.5 and Theorem 2.7. Making use of (1.5), (2.2), (2.5) and (2.15) and recalling that $\Gamma_{\zeta_{0}}$ is the fundamental solution of $\mathcal{H}_{\zeta_{0}}$, we have

$$
\begin{aligned}
& \left|u(x, t)-g\left(x_{0}\right)\right| \\
& \leq \int_{\mathbb{R}^{N}}\left|J\left(z ; \xi, T_{1}\right) g(\xi)\right| d \xi+\left|\int_{\mathbb{R}^{N}} \Gamma_{\left(x_{0}, T_{1}\right)}\left(z ; \xi, T_{1}\right) g(\xi) d \xi-g\left(x_{0}\right)\right| \\
& +\int_{\mathbb{R}^{N}}\left|\left(\Gamma_{\left(\xi, T_{1}\right)}\left(z ; \xi, T_{1}\right)-\Gamma_{\left(x_{0}, T_{1}\right)}\left(z ; \xi, T_{1}\right)\right) g(\xi)\right| d \xi \\
& \leq \mathbf{c}\left(t-T_{1}\right)^{\frac{\alpha}{2}} \int_{\mathbb{R}^{N}} \Gamma_{0}\left(\xi^{-1} \circ x, \mathbf{c}\left(t-T_{1}\right)\right)|g(\xi)| d \xi \\
& +\mathbf{c}\left(g, x_{0}\right) \int_{d(x, \xi) \leq \sigma} d\left(\xi, x_{0}\right)^{\frac{\alpha}{r}} \mathbf{E}\left(\xi^{-1} \circ x, \mathbf{c}\left(t-T_{1}\right)\right) d \xi \\
& +\mathbf{c}(M) \int_{d(x, \xi)>\sigma} 2 \mathbf{E}\left(\xi^{-1} \circ x, \mathbf{c}\left(t-T_{1}\right)\right) \exp \left(\mu d(\xi)^{2}\right) d \xi+o(1) \\
& \leq \mathbf{c}\left(t-T_{1}\right)^{\frac{\alpha}{2}}\left(\left|g\left(x_{0}\right)\right|+o(1)\right) \\
& +\mathbf{c}\left(g, x_{0}\right)\left(d\left(x, x_{0}\right)+\sigma\right)^{\frac{\alpha}{r}} \int_{\mathbb{R}^{N}} \Gamma_{0}\left(\xi^{-1} \circ x, \mathbf{c}\left(t-T_{1}\right)\right) d \xi \\
& +\mathbf{c}(M, \mu)\left(t-T_{1}\right)^{-\frac{Q}{2}} \int_{d(x, \xi)>\sigma} \exp \left(-\frac{d(x, \xi)^{2}}{\mathbf{c}\left(t-T_{1}\right)}\right) d \xi+o(1) \\
& =\mathbf{c}\left(g, x_{0}\right)(\sigma+o(1))^{\frac{\alpha}{r}}+\mathbf{c}(M, \mu) \int_{d(y)>\frac{\sigma}{\sqrt{t-T_{1}}}} \exp \left(-\mathbf{c} d(y)^{2}\right) d y+o(1),
\end{aligned}
$$

as $(x, t) \rightarrow\left(x_{0}, T_{1}\right)$.

Lemma 3.3. Let $\mu \geq 0$ and $T_{2}>T_{1}$ be such that $\left(T_{2}-T_{1}\right) \mu$ is small enough. Let $f(x, t)$ be a continuous function on $\mathbb{R}^{N} \times\left[T_{1}, T_{2}\right]$, locally Hölder continuous in $x$, uniformly w.r.t. $t$, satisfying the growth condition $|f(x, t)| \leq M \exp \left(\mu d(x)^{2}\right)$ for some constant $M>0$. Then the function

$$
V(z)=\int_{\mathbb{R}^{N} \times\left[T_{1}, t\right]} \Gamma_{\eta}(z ; \eta) f(\eta) d \eta, \quad z=(x, t) \in \mathbb{R}^{N} \times\left[T_{1}, T_{2}\right],
$$

belongs to the class $\mathfrak{C}^{2}\left(\mathbb{R}^{N} \times\left(T_{1}, T_{2}\right)\right) \cap C\left(\mathbb{R}^{N} \times\left[T_{1}, T_{2}\right]\right)$, and we have

$$
\mathcal{H} V(z)=-f(z)+\int_{\mathbb{R}^{N} \times\left[T_{1}, t\right]} Z_{1}(z ; \eta) f(\eta) d \eta, \quad z=(x, t) \in \mathbb{R}^{N} \times\left(T_{1}, T_{2}\right) .
$$


Proof. It is not difficult to prove that, given $B>0$, there exists $\delta>0$ such that the following estimate holds for every $b \in(0, B)$, provided $\left(T_{2}-T_{1}\right) \mu<\delta$ :

$$
\begin{aligned}
& \mathbf{E}\left(y^{-1} \circ x, b(t-s)\right) \exp \left(\mu d^{2}(y)\right) \\
& \quad \leq \mathbf{c} \mathbf{E}\left(y^{-1} \circ x, 2 b(t-s)\right) \exp \left(\mathbf{c} \mu d^{2}(x)\right), \quad 0<t-s \leq T_{2}-T_{1} .
\end{aligned}
$$

Using the estimate (3.3) and arguinig essentially as in the proof of Lemma 2.10 one can see that $V$ is well-posed, it has the required regularity, and

$$
\begin{aligned}
X_{j} V(z) & =\int_{\mathbb{R}^{N} \times\left[T_{1}, t\right]} X_{j} \Gamma_{\eta}\left(y^{-1} \circ x, t-s\right) f(\eta) y d \eta, \\
X_{i} X_{j} V(z) & =\lim _{\varepsilon \rightarrow 0^{+}} \int_{\mathbb{R}^{N} \times\left[T_{1}, t-\varepsilon\right]} X_{i} X_{j} \Gamma_{\eta}\left(y^{-1} \circ x, t-s\right) f(\eta) d \eta, \\
\partial_{t} V(z) & =f(z)+\lim _{\varepsilon \rightarrow 0^{+}} \int_{\mathbb{R}^{N} \times\left[T_{1}, t-\varepsilon\right]} \partial_{t} \Gamma_{\eta}\left(y^{-1} \circ x, t-s\right) f(\eta) d \eta .
\end{aligned}
$$

Recalling the definition (2.5) of $Z_{1}$, we obtain (3.2).

Lemma 3.4. Let $\mu \geq 0$ and $T_{2}>T_{1}$ be such that $\left(T_{2}-T_{1}\right) \mu$ is small enough. Let $f$ be a continuous function on $\mathbb{R}^{N} \times\left[T_{1}, T_{2}\right]$, satisfying the growth condition $|f(x, t)| \leq M \exp \left(\mu d(x)^{2}\right)$ for some constant $M>0$. Then the function

$$
\widetilde{f}(z)=\int_{\mathbb{R}^{N} \times\left[T_{1}, t\right]} \Phi(z ; \eta) f(\eta) d \eta, \quad z=(x, t) \in \mathbb{R}^{N} \times\left[T_{1}, T_{2}\right],
$$

is continuous on $\mathbb{R}^{N} \times\left[T_{1}, T_{2}\right]$. Moreover, $\widetilde{f}$ is locally Hölder continuous in $x$, uniformly w.r.t. $t$, and satisfies the growth condition $|\widetilde{f}(x, t)| \leq \widetilde{M} \exp \left(\mathbf{c} \mu d(x)^{2}\right)$ for some constant $\widetilde{M}>0$.

Proof. The growth estimate of $\widetilde{f}$ immediately follows from (2.10) and (3.3). The Hölder continuity easily follows from (2.11) and (3.3). We only need to prove that $\widetilde{f}(x, \cdot) \in C\left(\left[T_{1}, T_{2}\right]\right)$ for every fixed $x \in \mathbb{R}^{N}$. We have

$$
\begin{aligned}
& |\widetilde{f}(x, t+h)-\widetilde{f}(x, t)|=\int_{\mathbb{R}^{N} \times\left[T_{1}, t-\sigma\right]}|(\Phi(x, t+h ; \eta)-\Phi(x, t ; \eta)) f(\eta)| d \eta \\
& \quad+\int_{\mathbb{R}^{N} \times[t-\sigma, t+h]}|\Phi(x, t+h ; \eta) f(\eta)| d \eta+\int_{\mathbb{R}^{N} \times[t-\sigma, t]}|\Phi(x, t ; \eta) f(\eta)| d \eta .
\end{aligned}
$$

The first integral in the right-hand side vanishes as $h \rightarrow 0$, by dominated convergence, by making use of (2.10), (3.3) and Proposition 2.3. On the other hand, the other two integrals are small when $h$ vanishes and when $\sigma$ is small enough, again by means of (2.10) and (3.3).

Lemma 3.5. The statement of Theorem 3.1 holds if $g \equiv 0$.

Proof. We set $w=V_{f}+V_{\tilde{f}}$, where $\tilde{f}$ is defined by (3.4) and $V_{f}$ is defined by (3.1). From Lemmas 3.3 and 3.4 we have $w \in \mathfrak{C}^{2}\left(\mathbb{R}^{N} \times\left(T_{1}, T_{2}\right)\right) \cap C\left(\mathbb{R}^{N} \times\left[T_{1}, T_{2}\right]\right)$, and 
(using the estimates (2.7), (2.10) and (3.3))

$$
\begin{aligned}
\mathcal{H} w(z)= & -f(z)+\int_{\mathbb{R}^{N} \times\left[T_{1}, t\right]}\left(Z_{1}(z ; \zeta)-\Phi(z ; \zeta)\right) f(\zeta) d \zeta \\
& +\int_{\mathbb{R}^{N} \times\left[T_{1}, t\right]} Z_{1}(z ; \eta)\left(\int_{\mathbb{R}^{N} \times\left[T_{1}, s\right]} \Phi(\eta ; \zeta) f(\zeta) m y d \zeta\right) d \eta \\
= & -f(z)+\int_{\mathbb{R}^{N} \times\left[T_{1}, t\right]}\left(Z_{1}(z ; \zeta)-\Phi(z ; \zeta)\right. \\
& \left.+\int_{\mathbb{R}^{N} \times[\tau, t]} Z_{1}(z ; \eta) \Phi(\eta ; \zeta) d \eta\right) f(\zeta) d \zeta \\
= & -f(z),
\end{aligned}
$$

by means of Corollary 2.2. It is now sufficient to recognize that $w=u$ :

$$
\begin{aligned}
w(z) & =V_{f}(z)+\int_{\mathbb{R}^{N} \times\left[T_{1}, t\right]} f(\zeta)\left(\int_{\mathbb{R}^{N} \times[\tau, t]} \Gamma_{\eta}(z ; \eta) \Phi(\eta ; \zeta) d \eta\right) d \zeta \\
& =\int_{\mathbb{R}^{N} \times\left[T_{1}, t\right]} f(\zeta)\left(\Gamma_{\zeta}(z ; \zeta)+J(z ; \zeta)\right) d \zeta=u(z) .
\end{aligned}
$$

This ends the proof.

Proof of Theorem [3.1. It directly follows on collecting together Lemmas 3.2 and 3.5.

\section{LONG-Time ESTIMATES OF $\Gamma$}

We begin with the following weak maximum principle for $\mathcal{H}$ in the class $\mathfrak{C}^{2}$.

Theorem 4.1. Let $\Omega$ be a bounded open subset of $\mathbb{R}^{N+1}$ and let $t_{0} \in \mathbb{R}$. If $u \in$ $\mathfrak{C}^{2}(\Omega), \mathcal{H} u \geq 0$ in $\Omega \cap\left\{t<t_{0}\right\}$, and $\lim \sup u \leq 0$ in $\partial \Omega \cap\left\{t \leq t_{0}\right\}$, then $u \leq 0$ in $\Omega \cap\left\{t<t_{0}\right\}$.

Proof. The scheme of the proof is classical; the only difficulty is due to the "weak regularity" of $u$, namely $u \in \mathfrak{C}^{2}(\Omega)$. The thesis will follow if we show that the function $w_{\varepsilon}(x, t)=u(x, t)-\varepsilon /(T-t)$ satisfies $w_{\varepsilon} \leq 0$ in $\Omega_{T}:=\Omega \cap\{t<T\}$, for every $T<t_{0}$. To this end, we first prove that $w_{\varepsilon}$ has no maximum points in $\Omega_{T}$. Since $\left(a_{i, j}(z)\right)_{i, j}$ is positive definite and $\mathcal{H} w_{\varepsilon}>0$ by hypothesis, we only need to show that $\left(X_{i} X_{j} w_{\varepsilon}(\bar{z})\right)_{i, j} \leq 0$ (we remark that this matrix need not be symmetric), if $\bar{z}=(\bar{x}, \bar{t})$ is a maximum point of $w_{\varepsilon}$ in $\Omega_{T}$. Suppose, for a contradiction, that there exists $v \in \mathbb{R}^{m}$ such that $\sum_{i, j=1}^{m} X_{i} X_{j} w_{\varepsilon}(\bar{z}) v_{i} v_{j}>0$. We set $f(h)=w_{\varepsilon}(\bar{x} \circ \mu(h), \bar{t})$, where $\mu$ is the solution of $\dot{\mu}(h)=\sum_{j=1}^{m} v_{j} X_{j}(\mu(h)), \mu(0)=0$. We claim that

$$
\begin{gathered}
f \in C^{2}(-\sigma, \sigma) \quad \text { for some } \sigma>0, \\
\dot{f}(h)=\sum_{j=1}^{m} v_{j} X_{j} w_{\varepsilon}(\bar{x} \circ \mu(h), \bar{t}), \\
\ddot{f}(h)=\sum_{i, j=1}^{m} v_{i} v_{j} X_{i} X_{j} w_{\varepsilon}(\bar{x} \circ \mu(h), \bar{t}) .
\end{gathered}
$$

The proof of the claim directly follows from Lemma 4.2 below. This gives a contradiction, by taking the second order Taylor expansion of $f$ at zero: indeed, recalling that $\bar{z}$ is a maximum point of $w_{\varepsilon}$, we get

$$
w_{\varepsilon}(\bar{z}) \geq f(h)=f(0)+\ddot{f}(0) \frac{h^{2}}{2}(1+o(1))>f(0)=w_{\varepsilon}(\bar{z}), \quad \text { as } h \rightarrow 0 .
$$


Thus $w_{\varepsilon}$ has no maximum points in $\Omega_{T}$. The rest of the proof is classical (we stress that $u$ is continuous in the pair $(x, t)$, by the definition of $\left.\mathfrak{C}^{2}\right)$. Let $\bar{z} \in \bar{\Omega}_{T}$ be such that $\sup _{V \cap \Omega_{T}} w_{\varepsilon}=\sup _{\Omega_{T}} w_{\varepsilon}$ for every neighborhood $V$ of $\bar{z}$. By what we proved above, $\bar{z} \in \partial \Omega_{T}$. On the other hand, $\lim \sup w_{\varepsilon} \leq 0$ in $\partial \Omega_{T}$, by the definition of $w_{\varepsilon}$, by the continuity of $u$ and by the hypothesis on $\lim \sup u$. As a consequence, we get $w_{\varepsilon} \leq 0$ in $\Omega_{T}$. This completes the proof.

Lemma 4.2. Let $u$ be a continuous function on an open set $A \subseteq \mathbb{R}^{N}$, with continuous Lie derivatives along the vector fields $X_{1}, \ldots, X_{m}$ (in the pointwise sense (2.17)). Then, for every vector field $Z \in \operatorname{span}\left\{X_{1}, \ldots, X_{m}\right\}, Z=\sum_{j=1}^{m} v_{j} X_{j}$, and for every $x_{0} \in A$, the Lie derivative along $Z$ of $u$ at $x_{0}$ exists, and it is

$$
Z u\left(x_{0}\right)=\sum_{j=1}^{m} v_{j}\left(X_{j} u\right)\left(x_{0}\right) .
$$

Proof. By Remark 2.9, we know that $X_{1} u, \ldots, X_{m} u$ are derivatives also in the weak sense of distributions. We can define a $\mathbb{G}$-regularization $u_{\varepsilon}$ of $u$ (as in (2.21)) in such a way that $X_{j}\left(u_{\varepsilon}\right)=\left(X_{j} u\right)_{\varepsilon}$ and $u_{\varepsilon} \rightarrow u,\left(X_{j} u\right)_{\varepsilon} \rightarrow X_{j} u$, uniformly on the compact subsets of $A$ (see, e.g., [42]). Let $\mu$ be the solution of $\dot{\mu}=Z(\mu), \mu(0)=0$. Since $u_{\varepsilon}$ is smooth, for every small $h$ there exists a vanishing sequence $\varepsilon_{n}>0$ such that

$$
\frac{1}{h}\left(u_{\varepsilon_{n}}\left(x_{0} \circ \mu(h)\right)-u_{\varepsilon_{n}}\left(x_{0}\right)\right)=\sum_{j=1}^{m} v_{j} X_{j}\left(u_{\varepsilon_{n}}\right)\left(x_{0} \circ \mu\left(\theta\left(h, \varepsilon_{n}\right) h\right)\right),
$$

where $\theta\left(h, \varepsilon_{n}\right)$ converges to some $\theta(h) \in[0,1]$ as $n \rightarrow \infty$. Now, using the fact that $X_{j}\left(u_{\varepsilon_{n}}\right)=\left(X_{j} u\right)_{\varepsilon_{n}}$, first letting $n \rightarrow \infty$ and then letting $h \rightarrow 0$, we obtain the thesis.

The following version of the weak maximum principle in infinite strips easily follows from Theorem 4.1

Corollary 4.3. Let $u \in \mathfrak{C}^{2}\left(\mathbb{R}^{N} \times\left(T_{1}, T_{2}\right)\right)$ be such that $\mathcal{H} u \geq 0$ in $\mathbb{R}^{N} \times\left(T_{1}, T_{2}\right)$ and $\limsup u \leq 0$ in $\mathbb{R}^{N} \times\left\{T_{1}\right\}$ and at infinity. Then $u \leq 0$ in $\mathbb{R}^{N} \times\left(T_{1}, T_{2}\right)$.

From Corollary 4.3 we can immediately obtain some rough estimates of $\Gamma$.

Proposition 4.4. $\Gamma$ is a non-negative function. Moreover, $\sup _{t>\tau+1} \Gamma(x, t ; \xi, \tau)<$ $\infty$.

Proof. In order to prove the second assertion of the proposition, it is sufficient to notice that $M:=\sup _{t=\tau+1}|\Gamma(x, t ; \xi, \tau)|<\infty$ by means of (2.16), and then to apply Corollary 4.3 in the strip $\Omega=\mathbb{R}^{N} \times(\tau+1, \tau+T)$ to the function $u=\Gamma(\cdot ; \xi, \tau)-M$, observing that $u \in \mathfrak{C}^{2}(\bar{\Omega}), u \rightarrow-M$ at infinity and $\mathcal{H} u=0$ in $\Omega$, by (2.16) and Theorem 2.7.

We now prove that $\Gamma \geq 0$. We fix $x_{0} \in \mathbb{R}^{N}, t_{0}>\tau_{0}$, and we set $v=\Gamma\left(x_{0}, t_{0} ; \cdot \tau_{0}\right)$. Recalling Proposition 2.5. we only need to prove that $v \geq 0$ almost everywhere. To this end, we will show that $\int_{\mathbb{R}^{N}} v g \geq 0$, for every non-negative test function $g \in C_{0}^{\infty}\left(\mathbb{R}^{N}\right)$. From Theorem [3.1, it follows that $u(z)=\int_{\mathbb{R}^{N}} \Gamma\left(z ; \xi, \tau_{0}\right) g(\xi) d \xi$ belongs to the class $\mathfrak{C}^{2}\left(\left\{t>\tau_{0}\right\}\right), \mathcal{H} u=0$ in $\mathbb{R}^{N} \times\left(\tau_{0}, \infty\right)$ and $u \rightarrow g$ in $\mathbb{R}^{N} \times\left\{\tau_{0}\right\}$. Moreover, using the estimate (2.16), it is easy to see that $u$ goes to zero at infinity in the strip $\mathbb{R}^{N} \times\left(\tau_{0}, t_{0}+1\right)$. We now apply Corollary 4.3 and obtain $u \geq 0$ in $\mathbb{R}^{N} \times\left(\tau_{0}, t_{0}+1\right)$. In particular, we get $\int_{\mathbb{R}^{N}} v g=u\left(z_{0}\right) \geq 0$. 
We now prove the main result of this section.

Theorem 4.5. Fix $\lambda>\Lambda$ and $\varphi \in C_{0}^{\infty}\left(\mathbb{R}^{N}\right), 0 \leq \varphi \leq 1$. Set

$$
\widetilde{A}(x, t)=\varphi(x) A(x, t)+(1-\varphi(x)) \mathbb{I}_{m} / \lambda,
$$

where $\mathbb{I}_{m}$ denotes the $m \times m$ identity matrix. Then $\widetilde{A}$ satisfies (2.1), with $\widetilde{\Lambda}=\lambda$, $\widetilde{\alpha}=\alpha, \widetilde{k}=\mathbf{c}(\varphi)$. Hence, we can construct the fundamental solution $\widetilde{\Gamma}$ for the operator $\widetilde{\mathcal{H}}=\sum_{i, j=1}^{m} \widetilde{a}_{i, j}(x, t) X_{i} X_{j}-\partial_{t}$ (see Section 步). For every $K \Subset \mathbb{R}^{m}$ there exist positive constants $B=\mathbf{c}(K, \lambda, \varphi), M=\mathbf{c}(K, \lambda, \varphi)$ such that $\widetilde{\Gamma}$ satisfies the following estimate:

$$
\widetilde{\Gamma}(x, t ; \xi, \tau) \leq B(M+t-\tau)^{-m / 2} \exp \left(-\frac{\lambda\left|x^{(1)}-\xi^{(1)}\right|^{2}}{4(M+t-\tau)}\right),
$$

for every $(x, t),(\xi, \tau) \in \mathbb{R}^{N+1}$ such that $t>\tau+1$ and $\xi^{(1)} \in K$.

Proof. The proof of the first statement is straightforward. Let us prove the estimate (4.1). We set for brevity $w(x, t)=(M+t)^{-\frac{m}{2}} \exp \left(-\frac{\lambda}{4}\left|x^{(1)}\right|^{2} /(t+M)\right)$, where $M$ will be chosen later. We fix $\zeta_{0}=\left(\xi_{0}, \tau_{0}\right) \in \mathbb{R}^{N+1}$ such that $\xi_{0}^{(1)} \in K$, and we set $w_{0}(z)=w\left(z-\zeta_{0}\right)$. Observing that $X_{i} X_{j} w_{0}=\partial^{2} /\left(\partial x_{i}^{(1)} \partial x_{j}^{(1)}\right) w_{0}$, a direct computation gives

$$
\begin{aligned}
\widetilde{\mathcal{H}} w_{0}(x, t)=\frac{\lambda w_{0}(x, t)}{2\left(M+t-\tau_{0}\right)}( & \frac{\lambda\left\langle\widetilde{A}(x, t)\left(x^{(1)}-\xi_{0}^{(1)}\right), x^{(1)}-\xi_{0}^{(1)}\right\rangle-\left|x^{(1)}-\xi_{0}^{(1)}\right|^{2}}{2\left(M+t-\tau_{0}\right)} \\
& \left.+\frac{m}{\lambda}-\operatorname{trace}(\widetilde{A}(x, t))\right) .
\end{aligned}
$$

On the other hand, we have

$$
\begin{aligned}
& \langle\widetilde{A} v, v\rangle \leq \Lambda \varphi|v|^{2}+(1-\varphi)|v|^{2} \lambda^{-1}, \quad \forall v \in \mathbb{R}^{m}, \\
& \operatorname{trace}(\widetilde{A})=\varphi\left(\operatorname{trace}(A)-m \lambda^{-1}\right)+m \lambda^{-1} \geq m \varphi\left(\Lambda^{-1}-\lambda^{-1}\right)+m \lambda^{-1} .
\end{aligned}
$$

Hence, for $t>\tau_{0}$ we have

$$
\widetilde{\mathcal{H}} w_{0}(x, t) \leq \frac{\lambda w_{0}(x, t) \varphi(x)}{2\left(M+t-\tau_{0}\right)}\left(\frac{(\lambda \Lambda-1)\left|x^{(1)}-\xi_{0}^{(1)}\right|^{2}}{2\left(M+t-\tau_{0}\right)}-m\left(\Lambda^{-1}-\lambda^{-1}\right)\right) \leq 0
$$

if $M$ is chosen large enough, depending on $K, \lambda$ and $\varphi$. We now set

$$
\Omega_{0}=\left\{(x, t) \in \mathbb{R}^{N+1}: t>\tau_{0}\right\} \backslash\left\{d\left(x, \xi_{0}\right) \leq 1,\left|t-\tau_{0}\right| \leq 1\right\} .
$$

We have $\widetilde{\mathcal{H}} w_{0} \leq 0, \widetilde{\mathcal{H}}\left(\widetilde{\Gamma}\left(\cdot ; \zeta_{0}\right)\right)=0$ in $\Omega_{0}$ (see Theorem 2.7). Moreover, $w_{0}$ is bounded from below in $\partial \Omega_{0} \backslash\left\{t=\tau_{0}\right\}$, by a constant depending only on $M$ and $\lambda$ (but not on $\zeta_{0}$ ). Furthermore, from (2.16) it follows that $\widetilde{\Gamma}\left(\cdot ; \zeta_{0}\right)$ is bounded in $\partial \Omega_{0} \backslash\left\{t=\tau_{0}\right\}$, by a constant depending only on $\lambda$ and $\varphi$ (and not on $\zeta_{0}$ ), and that $\widetilde{\Gamma}\left(\cdot ; \zeta_{0}\right)$ vanishes in $\partial \Omega_{0} \cap\left\{t=\tau_{0}\right\}$ and at infinity on every strip. Therefore, using Theorem 4.1, we can obtain the desired estimate (4.1) in $\Omega_{0}$.

In order to complete the proof of Theorem [1.2, we shall use the following a priori Schauder-type estimates. 
Proposition 4.6. Let $\Omega$ be an open subset of $\mathbb{R}^{N}$ and $0<\beta \leq \alpha$. Suppose the coefficients $a_{i, j}(x)$ of $\mathcal{L}$ are smooth. Then, for every $u \in \Gamma_{\text {loc }}^{2+\beta}(\Omega)$ and for every bounded domain $O \subset \bar{O} \subset \Omega$, we have

$$
\|u\|_{\Gamma^{2+\beta}(\bar{O})} \leq \mathbf{c}(O, \Omega, \beta, \Lambda, k, \alpha)\left(\sup _{\Omega}|u|+\|\mathcal{L} u\|_{\Gamma^{\beta}(\Omega)}\right),
$$

where $\Lambda, k, \alpha$ have been introduced in (2.1).

If $\Omega \subseteq \mathbb{R}^{N+1}$ and the coefficients $a_{i, j}(x, t)$ of $\mathcal{H}$ are smooth, inequality (4.2) holds with $\mathcal{L}$ replaced by $\mathcal{H}$ (and with the related "parabolic" meaning of $\Gamma^{2+\beta}$ ).

Proof. Inequality (4.2) follows from the result of Xu [44] (see also the recent paper by Capogna and Han [13, where a deeper result is proved for our operator $\mathcal{L})$. The techniques used in [44] are suitable to handle the parabolic operator $\mathcal{H}$ too. We omit the details.

Remark 4.7. Let $O$ be a bounded domain of $\mathbb{R}^{N+1}$ and let $0<\beta^{\prime}<\beta<1$. Then any bounded sequence in $\Gamma^{2+\beta}(\bar{O})$ admits a convergent subsequence in $\Gamma^{2+\beta^{\prime}}(\bar{O})$. An analogous result holds for $O \subset \mathbb{R}^{N}$ (with the corresponding meaning of $\Gamma^{2+\beta}$ ).

Corollary 4.8. Suppose the coefficients $a_{i, j}$ of $\mathcal{H}$ are smooth. Let $\Omega \subseteq \mathbb{R}^{N+1}$ and $u \in \mathfrak{C}^{2}(\Omega)$ be such that $\mathcal{H} u \in \Gamma_{\text {loc }}^{\beta}(\Omega)$. Then $u \in \Gamma_{\text {loc }}^{2+\beta}(\Omega)$.

Proof. We set $f=\mathcal{H} u$ and define a sequence of smooth functions $\left\{f_{j}\right\}_{j}$ obtained as "G-G-regularization" of $f$ as in (4.5) below. Then it is not difficult to see that

$$
\sup _{j}\left\|f_{j}\right\|_{\Gamma^{\beta}(K)}<\infty
$$

for every $K \Subset \Omega$, and that $\left\{f_{j}\right\}_{j}$ is uniformly convergent to $f$ on the compact subsets of $\Omega$. Since $A=\left(a_{i, j}\right)_{i, j}$ is positive-definite and $C^{\infty}$, there exists a symmetric matrix $B=\left(b_{i, j}\right)_{i, j}$ with $C^{\infty}$ entries such that $B^{2}=A$. Then, letting $Y_{i}=$ $\sum_{j=1}^{m} b_{i, j}(x, t) X_{j}(i=1, \ldots, m)$, we can write $\mathcal{H}$ as follows:

$$
\mathcal{H}=\sum_{j=1}^{m} Y_{j}^{2}-\partial_{t}-Y_{0}
$$

where $Y_{0}=\sum_{i=1}^{m}\left(\sum_{j=1}^{m} Y_{j} b_{j, i}(x, t)\right) X_{i}$. Moreover, the family $\left\{Y_{1}, \ldots, Y_{m}, \partial_{t}+Y_{0}\right\}$ satisfies at any point $(x, t)$ the Hörmander hypoellipticity condition

$$
\operatorname{rank}\left(\operatorname{Lie}\left\{Y_{1}, \ldots, Y_{m}, \partial_{t}+Y_{0}\right\}(x, t)\right)=N+1 \text {. }
$$

Then, by a classical result by Bony [6], there exists a basis $\mathcal{V}$ of open sets $V \Subset \Omega$ such that the Dirichlet problem

$$
\mathcal{H} \omega=f_{j} \text { in } V,\left.\quad \omega\right|_{\partial V}=\left.u\right|_{\partial V}
$$

has a solution $u_{j} \in C^{\infty}(V) \cap C(\bar{V})$. By Proposition 4.6] by (4.3), by Remark 4.7 and by the maximum principle for $\mathcal{H}$, up to a subsequence, $\left\{u_{j}\right\}_{j}$ converges in $\Gamma_{\text {loc }}^{2+\beta^{\prime}}(V)$ (for every $0<\beta^{\prime}<\beta$ ) to a function $v \in \Gamma_{\text {loc }}^{2+\beta}(V) \cap C(\bar{V})$ such that $\mathcal{H} v=f$ in $V$ and $\left.v\right|_{\partial V}=\left.u\right|_{\partial V}$. We explicitly remark that $v \in \Gamma_{\text {loc }}^{2+\beta}(V)$, since the norms of $v$ in $\Gamma_{\text {loc }}^{2+\beta^{\prime}}$ are uniformly bounded in $\beta^{\prime} \in(0, \beta)$ (while $v \in C(\bar{V})$ follows from the existence of suitable barriers for $V$ as in $[\underline{6}$ ). From the maximum principle for $\mathfrak{C}^{2}(V)$-functions (see Theorem 4.1), we obtain $u=v$ in $V$, and the assertion follows since $\mathcal{V}=\{V\}$ is a basis of $\Omega$.

Corollary 4.9. Suppose the coefficients $a_{i, j}$ of $\mathcal{H}$ are smooth. Let $f \in \Gamma_{\text {loc }}^{\beta}(\Omega), \Omega \subseteq$ $\mathbb{R}^{N+1}$ and $u \in C(\Omega)$ be such that $\mathcal{H} u=f$ in $\Omega$ in the weak sense of distributions. Then $u \in \Gamma_{\text {loc }}^{2+\beta}(\Omega)$. An analogous result holds for $\mathcal{L}$. 
Proof. We use the notation of Corollary 4.8, Let $V \in \mathcal{V}$ and let $v \in \Gamma_{\text {loc }}^{2+\beta}(V) \cap$ $C(\bar{V})$ be such that $\mathcal{H} v=f$ in $V$ and $\left.v\right|_{\partial V}=\left.u\right|_{\partial V}$. Then, in the weak sense of distributions, $\mathcal{H}(u-v)=0$ in $V$. Moreover, $u-v=0$ on $\partial V$. Since $\mathcal{H}$ is hypoelliptic, it follows that $u-v \in C^{\infty}(V)$ and, by the maximum principle, $u=v$ in $V$. The assertion follows, since $\mathcal{V}=\{V\}$ is a basis of $\Omega$.

We finally complete the proof of Theorem 1.2 .

Proof of Theorem 1.2. Thanks to Proposition 2.5. Theorem 2.7 and Proposition 4.4, in order to complete the proof of (i) and (ii), it only remains to show that

$$
\Gamma(\cdot ; \zeta) \in \Gamma_{\text {loc }}^{2+\alpha}\left(\mathbb{R}^{N+1} \backslash\{\zeta\}\right) .
$$

To this end, we shall approximate $\mathcal{H}$ by suitable smooth coefficient operators $\mathcal{H}^{\varepsilon}$. Let $\Psi \in C_{0}^{\infty}\left(B_{d}(0,1) \times(-1,1)\right)$ be such that $0 \leq \Psi(x, t)=\Psi\left(x^{-1}, t\right), \int \Psi=1$, and set $\Psi^{\varepsilon}(x, t)=\varepsilon^{-Q-2} \Psi\left(\delta_{\varepsilon^{-1}} x, \varepsilon^{-2} t\right)$. We define

$$
a_{i, j}^{\varepsilon}(x, t)=\int_{\mathbb{R}^{N+1}} \Psi^{\varepsilon}\left(y \circ x^{-1}, s-t\right) a_{i, j}(y, s) d y d s .
$$

Such a definition of regularization allows to prove that the coefficients $a_{i, j}^{\varepsilon}$, besides being smooth, satisfy (2.11) (with the same $\Lambda, \alpha$ and $k$ ) and converge to $a_{i, j}$, uniformly on the compact subsets of $\mathbb{R}^{N+1}$, as $\varepsilon \rightarrow 0^{+}$. Hence, we can construct the fundamental solution $\Gamma^{\varepsilon}$ of the operator $\mathcal{H}^{\varepsilon}=\sum_{i, j=1}^{m} a_{i, j}^{\varepsilon}(x, t) X_{i} X_{j}-\partial_{t}$, as in Section 2. We claim that

$$
\Gamma^{\varepsilon}(z ; \zeta) \longrightarrow \Gamma(z ; \zeta), \quad \text { as } \varepsilon \rightarrow 0^{+}, \text {for all } z, \zeta \in \mathbb{R}^{N+1} .
$$

Moreover, by Theorem 2.7 and Corollary $4.8 \Gamma^{\varepsilon}(\cdot ; \zeta) \in \Gamma_{\text {loc }}^{2+\alpha}\left(\mathbb{R}^{N+1} \backslash\{\zeta\}\right)$ and $\mathcal{H}^{\varepsilon}\left(\Gamma^{\varepsilon}(\cdot ; \zeta)\right)=0$ in $\mathbb{R}^{N+1} \backslash\{\zeta\}$. We also recall the estimate (2.16) of $\Gamma^{\varepsilon}$, which does not depend on $\varepsilon$, since the $a_{i, j}^{\varepsilon}$ satisfy (2.1) with the same $\Lambda, \alpha$ and $k$, fixed at the beginning of Section 2 Now, using Proposition 4.6. we obtain

$$
\left\|\Gamma^{\varepsilon}(\cdot ; \zeta)\right\|_{\Gamma^{2+\alpha}(K)} \leq \mathbf{c}(K, \Omega) \sup _{\Omega}\left|\Gamma^{\varepsilon}(\cdot ; \zeta)\right| \leq \mathbf{c}(K, \Omega, \zeta),
$$

for every $K=\bar{O} \Subset \Omega \Subset \mathbb{R}^{N} \backslash\{\zeta\}$ and $\varepsilon>0$. Recalling Remark 4.7, we infer that, for some vanishing sequence $\varepsilon_{j}>0, \Gamma^{\varepsilon_{j}}(\cdot ; \zeta)$ converges in $\Gamma^{2+\beta}(K)$, as $j \rightarrow \infty$, for every $K=\bar{O} \Subset \mathbb{R}^{N+1} \backslash\{\zeta\}$ and every $\beta \in(0, \alpha)$. By means of (4.6), the limit must necessarily be $\Gamma(\cdot ; \zeta)$. Therefore, we have proved that (4.4) holds (we use here the fact that the norms of $\Gamma(\cdot ; \zeta)$ in $\Gamma_{\text {loc }}^{2+\beta}$ are uniformly bounded in $\left.\beta \in(0, \alpha)\right)$ and that for every $\zeta \in \mathbb{R}^{N+1}$ there exists a vanishing sequence $\varepsilon_{j}$ such that

$$
\Gamma^{\varepsilon_{j}}(\cdot ; \zeta) \stackrel{j \rightarrow \infty}{\longrightarrow} \Gamma(\cdot ; \zeta) \quad \text { in } \Gamma^{2+\beta}(K), \quad \forall K=\bar{O} \Subset \mathbb{R}^{N+1} \backslash\{\zeta\}, \quad \forall \beta \in(0, \alpha) .
$$

In order to prove the claimed (4.6), we follow the steps in the construction of the fundamental solution $\Gamma$ in Section 2, exploiting the fact that all the constants c in the estimates used there do not depend on $\varepsilon$, but only on the fixed parameters $\Lambda, \alpha$ and $k$. We first observe that $X_{i_{1}} \ldots X_{i_{p}}\left(\partial_{t}\right)^{q} \Gamma_{\zeta_{0}}^{\varepsilon}$ converges pointwise to $X_{i_{1}} \ldots X_{i_{p}}\left(\partial_{t}\right)^{q} \Gamma_{\zeta_{0}}$, as $\varepsilon \rightarrow 0^{+}$, by means of Theorem 1.1. Using the estimate (2.9), it is then easy to prove, by induction, that $Z_{n}^{\varepsilon}$ converges pointwise to $Z_{n}$ for every $n \in \mathbb{N}$, and that the series which defines $\Phi^{\varepsilon}$ is convergent, uniformly in $\varepsilon \geq 0$. Hence also $\Phi^{\varepsilon}$ converges pointwise to $\Phi$. The convergence of $J^{\varepsilon}$ to $J$ easily follows by dominated convergence (recalling the estimates (2.2) and (2.10), which are uniform in $\varepsilon$ ) and straightforwardly gives (4.6). 
We now complete the proof of (iii) and (iv). From Theorem 3.1 and Corollary 4.8, it immediately follows that the statements (iii) and (iv) hold for $\mathcal{H}^{\varepsilon}$. In order to prove them for $\mathcal{H}$, we again use (4.6), Proposition 4.6] and Remark 4.7, as in the proof of (4.4).

We also prove the following result, which will be used in Section 5 .

Proposition 4.10. Suppose that the coefficients $a_{i, j}$ of $\mathcal{H}$ are smooth, so that the adjoint operator $\mathcal{H}^{*}$ is defined. Then, for every fixed $\zeta \in \mathbb{R}^{N+1}$, we have

$$
\mathcal{H}(\Gamma(\cdot ; \zeta))=-\delta_{\zeta}
$$

(the Dirac measure supported at $\{\zeta\})$. In other words,

$$
\int_{\mathbb{R}^{N+1}} \Gamma(z ; \zeta) \mathcal{H}^{*} \psi(z) d z=-\psi(\zeta)
$$

for every test function $\psi \in C_{0}^{\infty}\left(\mathbb{R}^{N+1}\right)$.

Proof. Using (2.16) once again, we immediately see that

$$
\int_{\mathbb{R}^{N+1} \times \mathbb{R}^{N+1}}\left|\varphi(\zeta) \Gamma(z ; \zeta) \mathcal{H}^{*} \psi(z)\right| d z d \zeta<\infty
$$

for every test function $\varphi \in C_{0}^{\infty}\left(\mathbb{R}^{N+1}\right)$. Setting $I(\zeta)=\int \Gamma(z ; \zeta) \mathcal{H}^{*} \psi(z) d z$, we have

$$
\int \varphi I=\int \mathcal{H}^{*} \psi(z)\left(\int \varphi(\zeta) \Gamma(z ; \zeta) d \zeta\right) d z=-\int \varphi \psi,
$$

by means of Theorem 1.2 (iii) (see also Remark 2.9). Hence $I=-\psi$ almost everywhere. It is now sufficient to prove that $I$ is continuous. To this end, one can see that $I$ is the uniform limit, as $\sigma \rightarrow 0^{+}$, of the continuous functions

$$
\zeta \mapsto \int_{\mathbb{R}^{N} \times[\tau+\sigma, \infty)} \Gamma(z ; \zeta) \mathcal{H}^{*} \psi(z) d z
$$

by using Proposition 2.5 ,

\section{Construction of the fundamental solution for $\mathcal{L}$}

The aim of this section is to prove Theorem 1.5. Throughout the section, we shall always suppose that $m>2$ and that the coefficients $a_{i, j}$, introduced in (2.1), depend only on $x \in \mathbb{R}^{N}$. Moreover, $\Omega$ will always denote a fixed bounded open subset of $\mathbb{R}^{N}$, and $\varphi$ a fixed $C_{0}^{\infty}$-function on $\mathbb{R}^{N}$ such that $0 \leq \varphi \leq 1$ and $\varphi \equiv 1$ in $\Omega$. We define the $\mathbb{G}$-regularization $A^{\varepsilon}(x)=\left(a_{i, j}^{\varepsilon}(x)\right)_{i, j}$ as in (2.21), so that the $a_{i, j}^{\varepsilon}$, besides being smooth, satisfy (2.1) (with the same $\Lambda, \alpha$ and $k$ ) and converge to $a_{i, j}$, uniformly on the compact subsets of $\mathbb{R}^{N}$, as $\varepsilon \rightarrow 0^{+}$(see also the proof of Theorem (1.2). We set

$$
\widetilde{A}^{\varepsilon}(x)=\varphi(x) A^{\varepsilon}(x)+(1-\varphi(x))(4 \Lambda)^{-1} \mathbb{I}_{m}
$$

(where $\mathbb{I}_{m}$ denotes the $m \times m$ identity matrix) and we define

$$
\widetilde{\mathcal{H}}^{\varepsilon}=\widetilde{\mathcal{L}}^{\varepsilon}-\partial_{t}=\sum_{i, j=1}^{m} \widetilde{a}_{i, j}^{\varepsilon}(x) X_{i} X_{j}-\partial_{t} .
$$

We also define $\widetilde{A}=\varphi A+(1-\varphi)(4 \Lambda)^{-1} \mathbb{I}_{m}$ and $\widetilde{\mathcal{L}}, \widetilde{\mathcal{H}}$ accordingly. By means of Theorem 4.5. $\widetilde{A}, \widetilde{A}_{\varepsilon}$ satisfy (2.1), with $\widetilde{\Lambda}=4 \Lambda, \widetilde{\alpha}=\alpha, \widetilde{k}=\mathbf{c}(\varphi)$ (not depending 
on $\varepsilon$ ), and the fundamental solutions $\widetilde{\Gamma}, \widetilde{\Gamma}^{\varepsilon}$ of $\widetilde{\mathcal{H}}, \widetilde{\mathcal{H}}^{\varepsilon}$ satisfy the following estimate for every $K \Subset \mathbb{R}^{m}$ :

$$
\begin{aligned}
\widetilde{\Gamma}(x, t ; \xi, \tau), & \widetilde{\Gamma}^{\varepsilon}(x, t ; \xi, \tau) \\
& \leq \mathbf{c}_{1}(K, \varphi)\left(\mathbf{c}_{2}(K, \varphi)+t-\tau\right)^{-m / 2} \exp \left(-\frac{\Lambda\left|x^{(1)}-\xi^{(1)}\right|^{2}}{\mathbf{c}_{2}(K, \varphi)+t-\tau}\right)
\end{aligned}
$$

for every $(x, t),(\xi, \tau) \in \mathbb{R}^{N+1}$ such that $t>\tau+1$ and $\xi^{(1)} \in K$. Moreover, $\widetilde{A}_{\varepsilon}$ converges uniformly to $\widetilde{A}$, as $\varepsilon \rightarrow 0^{+}$, on the compact subsets of $\mathbb{R}^{N}$. We now set

$$
\begin{aligned}
& \widetilde{\gamma}(x, \xi)=\int_{0}^{\infty} \widetilde{\Gamma}(x, t ; \xi, 0) d t, \\
& \widetilde{\gamma}^{\varepsilon}(x, \xi)=\int_{0}^{\infty} \widetilde{\Gamma}^{\varepsilon}(x, t ; \xi, 0) d t, \quad x \neq \xi \in \mathbb{R}^{N} .
\end{aligned}
$$

From (2.16), (5.1) and Proposition 4.4 it follows that the integrals in (5.2) are convergent and, for every $K \Subset \mathbb{R}^{m}$,

$$
\begin{aligned}
0 \leq & \widetilde{\gamma}(x, \xi), \widetilde{\gamma}^{\varepsilon}(x, \xi) \leq \mathbf{c}(\varphi) \int_{0}^{1} t^{-Q / 2} \exp \left(-\frac{d(x, \xi)^{2}}{\mathbf{c} t}\right) d t \\
& +\mathbf{c}_{1}(K, \varphi) \int_{1}^{\infty}\left(\mathbf{c}_{2}(K, \varphi)+t\right)^{-m / 2} \exp \left(-\frac{\Lambda\left|x^{(1)}-\xi^{(1)}\right|^{2}}{\mathbf{c}_{2}(K, \varphi)+t}\right) d t \\
\leq & \mathbf{c}(\varphi) d(x, \xi)^{2-Q}+\mathbf{c}(K, \varphi) \min \left\{1,\left|x^{(1)}-\xi^{(1)}\right|^{2-m}\right\}, \quad \xi^{(1)} \in K .
\end{aligned}
$$

From the same estimates (2.16), (5.1) and the continuity of $\widetilde{\Gamma}, \widetilde{\Gamma}^{\varepsilon}$ (see Proposition 2.5), it follows (by dominated convergence) that $\widetilde{\gamma}, \widetilde{\gamma}^{\varepsilon}$ are continuous functions away from the diagonal of $\mathbb{R}^{N} \times \mathbb{R}^{N}$.

Lemma 5.1. For every $\xi \in \mathbb{R}^{N}$, we have

$$
\widetilde{\mathcal{L}}^{\varepsilon}\left(\widetilde{\gamma}^{\varepsilon}(\cdot, \xi)\right)=-\delta_{\xi}
$$

(the Dirac measure supported at $\xi$ ). Moreover, for every $\psi \in C_{0}^{\infty}\left(\mathbb{R}^{N}\right)$, the function

$$
w(x)=\int_{\mathbb{R}^{N}} \widetilde{\gamma}^{\varepsilon}(x, \xi) \psi(\xi) d \xi
$$

belongs to the class $\Gamma_{\text {loc }}^{2+\alpha}\left(\mathbb{R}^{N}\right)$, and $\widetilde{\mathcal{L}}^{\varepsilon} w=-\psi$ in $\mathbb{R}^{N}$.

Proof. Let $\psi \in C_{0}^{\infty}\left(\mathbb{R}^{N}\right)$ and set $\psi_{n}(x, t)=\psi(x) \theta(|t| / n)$, where $\theta$ is a smooth cutoff function defined on $\mathbb{R}$, such that $\theta(s)=1$ for $s \leq 1, \theta(s)=0$ for $s \geq 2$. From Proposition 4.10, it follows that

$$
\begin{aligned}
-\psi(\xi) & =-\psi_{n}(\xi, 0)=\int_{\mathbb{R}^{N+1}} \widetilde{\Gamma}^{\varepsilon}(x, t ; \xi, 0)\left(\widetilde{\mathcal{H}}^{\varepsilon}\right)^{*} \psi_{n}(x, t) d x d t \\
& \stackrel{n \rightarrow \infty}{\longrightarrow} \int_{\mathbb{R}^{N+1}} \widetilde{\Gamma}^{\varepsilon}(x, t ; \xi, 0)\left(\widetilde{\mathcal{L}}^{\varepsilon}\right)^{*} \psi(x) d x d t \\
& =\int_{\mathbb{R}^{N}} \widetilde{\gamma}^{\varepsilon}(x, \xi)\left(\widetilde{\mathcal{L}}^{\varepsilon}\right)^{*} \psi(x) d x,
\end{aligned}
$$

by dominated convergence (recalling (5.3)). This proves (5.4). Using the continuity of $\widetilde{\gamma}^{\varepsilon}$, one can see that the function $w$ defined in (5.5) is continuous on $\mathbb{R}^{N}$, 
approximating $w$ uniformly with the continuous functions

$$
w_{n}(x)=\int_{\mathbb{R}^{N}} \widetilde{\gamma}^{\varepsilon}(x, \xi)(1-\theta(n d(x, \xi))) \psi(\xi) d \xi .
$$

Indeed, by (5.3), we have

$$
\begin{aligned}
\sup _{\mathbb{R}^{N}}\left|w_{n}-w\right| & \leq \mathbf{c}(\varphi, \psi, \theta) \sup _{x \in \mathbb{R}^{N}} \int_{d(x, \xi) \leq \frac{2}{n}}\left(1+d(x, \xi)^{2-Q}\right) d \xi \\
& =\mathbf{c}(\varphi, \psi, \theta) \int_{d(y) \leq \frac{2}{n}}\left(1+d(y)^{2-Q}\right) d y \longrightarrow 0, \quad \text { as } n \rightarrow \infty .
\end{aligned}
$$

Moreover, $\widetilde{\mathcal{L}}^{\varepsilon} w=-\psi$ on $\mathbb{R}^{N}$, in the weak sense of distributions, since (5.4) gives

$$
\begin{aligned}
\int w\left(\widetilde{\mathcal{L}}^{\varepsilon}\right)^{*} f & =\int\left(\int \widetilde{\gamma}^{\varepsilon}(x, \xi) \psi(\xi) d \xi\right)\left(\widetilde{\mathcal{L}}^{\varepsilon}\right)^{*} f(x) d x \\
& =\int \psi(\xi)\left(\int \widetilde{\gamma}^{\varepsilon}(x, \xi)\left(\widetilde{\mathcal{L}}^{\varepsilon}\right)^{*} f(x) d x\right) d \xi=-\int \psi f
\end{aligned}
$$

for every test function $f \in C_{0}^{\infty}\left(\mathbb{R}^{N}\right)$. In order to complete the proof, it is now sufficient to recall Corollary 4.9 and Remark 2.9 ,

Theorem 5.2. For every $\xi \in \mathbb{R}^{N}$ we have

$$
\widetilde{\gamma}(\cdot, \xi) \in \Gamma_{\text {loc }}^{2+\alpha}\left(\mathbb{R}^{N} \backslash\{\xi\}\right) \text { and } \widetilde{\mathcal{L}}(\widetilde{\gamma}(\cdot, \xi))=0 \text { in } \mathbb{R}^{N} \backslash\{\xi\} .
$$

Moreover, for every $\psi \in C_{0}^{\infty}\left(\mathbb{R}^{N}\right)$, the function

$$
w(x)=\int_{\mathbb{R}^{N}} \widetilde{\gamma}(x, \xi) \psi(\xi) d \xi
$$

belongs to the class $\Gamma_{\text {loc }}^{2+\alpha}\left(\mathbb{R}^{N}\right)$, and we have $\widetilde{\mathcal{L}} w=-\psi$ in $\mathbb{R}^{N}$.

Proof. We first observe that, by means of (4.6), $\widetilde{\gamma}^{\varepsilon}$ converges pointwise to $\widetilde{\gamma}$, as $\varepsilon \rightarrow 0^{+}$, by dominated convergence (see also $(5.3)$ ). Moreover, recalling that $\widetilde{\gamma}^{\varepsilon}(\cdot, \xi)$ is continuous away from $\xi$, that (5.4) holds and that the coefficients of $\widetilde{\mathcal{L}}^{\varepsilon}$ are smooth, we can use Corollary 4.9 and obtain that $\widetilde{\gamma}^{\varepsilon}(\cdot, \xi) \in \Gamma_{\text {loc }}^{2+\alpha}\left(\mathbb{R}^{N} \backslash\{\xi\}\right)$, jointly with $\widetilde{\mathcal{L}}^{\varepsilon}\left(\widetilde{\gamma}^{\varepsilon}(\cdot, \xi)\right)=0$ in $\mathbb{R}^{N} \backslash\{\xi\}$. Using Proposition 4.6 together with Remark 4.7 and the estimate (5.3), one can prove that, for some vanishing sequence $\varepsilon_{j}, \widetilde{\gamma}^{\varepsilon_{j}}(\cdot, \xi)$ converges in $\Gamma^{2+\beta}(K)$, as $j \rightarrow \infty$, for every $K=\bar{O} \Subset \mathbb{R}^{N} \backslash\{\xi\}$ and for every $\beta \in(0, \alpha)$. On the other hand, the limit must necessarily be $\widetilde{\gamma}(\cdot, \xi)$, since $\widetilde{\gamma}^{\varepsilon} \rightarrow \widetilde{\gamma}$ pointwise. Therefore, we get $\widetilde{\gamma}(\cdot, \xi) \in \Gamma_{\text {loc }}^{2+\alpha}\left(\mathbb{R}^{N} \backslash\{\xi\}\right)$ (we use here the fact that the norms of $\widetilde{\gamma}(\cdot ; \xi)$ in $\Gamma_{\text {loc }}^{2+\beta}$ are uniformly bounded in $\left.\beta \in(0, \alpha)\right)$ and $\widetilde{\mathcal{L}}(\widetilde{\gamma}(\cdot, \xi))=0$ in $\mathbb{R}^{N} \backslash\{\xi\}$. With the same arguments, we can prove the last statement of Theorem 5.2, starting from Lemma 5.1 and observing that

$$
w_{\varepsilon}(x)=\int_{\mathbb{R}^{N}} \widetilde{\gamma}^{\varepsilon}(x, \xi) \psi(\xi) d \xi \longrightarrow \int_{\mathbb{R}^{N}} \widetilde{\gamma}(x, \xi) \psi(\xi) d \xi, \quad \text { as } \varepsilon \rightarrow 0^{+},
$$


by dominated convergence (see also (5.3)). We explicitly remark that from (5.3) it follows that (setting $F=\left\{x \in \mathbb{R}^{N} \mid d(x, \operatorname{supp}(\psi)) \leq 1\right\}$ )

$$
\begin{aligned}
\sup _{\mathbb{R}^{N}}\left|w_{\varepsilon}\right| \leq \mathbf{c}(\varphi, \psi) \sup _{x \in \mathbb{R}^{N}} \int_{\operatorname{supp}(\psi)}\left(1+d(x, \xi)^{2-Q}\right) d \xi \\
\leq \mathbf{c}(\varphi, \psi)\left(\sup _{x \in F} \int_{(\operatorname{supp}(\psi))^{-1} \circ F}\left(1+d(y)^{2-Q}\right) d y\right. \\
\left.\quad+\sup _{x \notin F} \int_{\operatorname{supp}(\psi)} d \xi\right) \leq \mathbf{c}(\varphi, \psi) .
\end{aligned}
$$

This allows us to prove (by Proposition 4.6) that $w_{\varepsilon}$ is bounded in $\Gamma^{2+\alpha}(K)$ for any $K=\bar{O} \Subset \mathbb{R}^{N}$.

Proof of Theorem 1.5. Theorem 1.5 is a straightforward consequence of the results in this section, setting $\gamma=\widetilde{\gamma}$ and observing that $\mathcal{L}=\widetilde{\mathcal{L}}$ in $\Omega$ by definition.

\section{REFERENCES}

1. G. K. Alexopoulos, Sub-Laplacians with drift on Lie groups of polynomial volume growth, Mem. Amer. Math. Soc., 155 (2002), no. 739. MR 2003c:22015

2. A. Bonfiglioli, E. Lanconelli, and F. Uguzzoni, Uniform Gaussian estimates of the fundamental solutions for heat operators on Carnot groups, Adv. Differential Equations 7 (2002), 1153-1192.

3. A. Bonfiglioli and F. Uguzzoni, Families of diffeomorphic sub-Laplacians and free Carnot groups, to appear in Forum Math.

4. A. Bonfiglioli and F. Uguzzoni, A note on lifting of Carnot groups, preprint.

5. A. Bonfiglioli and F. Uguzzoni, Harnack inequality for non-divergence form operators on stratified groups, preprint.

6. J.-M. Bony, Principe du maximum, inégalité de Harnack et unicité du problème de Cauchy pour les opérateurs elliptiques dégénérés, Ann. Inst. Fourier (Grenoble) 19 (1969), 277-304. MR 41:7486

7. M. Bramanti and L. Brandolini, $L^{p}$ estimates for nonvariational hypoelliptic operators with VMO coefficients, Trans. Amer. Math. Soc. 352 (2000), no. 2, 781-822. MR 2000c:35026

8. M. Bramanti and L. Brandolini, $L^{p}$ estimates for uniformly hypoelliptic operators with discontinuous coefficients on homogeneous groups, to appear in Rend. Sem. Mat. Univ. Politec. Torino.

9. L. Capogna, Regularity of quasi-linear equations in the Heisenberg group, Comm. Pure Appl. Math. 50 (1997), no. 9, 867-889. MR 98k:22037

10. L. Capogna, Regularity for quasilinear equations and 1-quasiconformal maps in Carnot groups, Math. Ann. 313 (1999), no. 2, 263-295. MR 2000a:35027

11. L. Capogna, D. Danielli, and N. Garofalo, An embedding theorem and the Harnack inequality for nonlinear subelliptic equations Comm. Partial Differential Equations 18 (1993), no. 9-10, 1765-1794. MR 94j:35038

12. L. Capogna, D. Danielli, and N. Garofalo, Capacitary estimates and the local behavior of solutions of nonlinear subelliptic equations, Amer. J. Math. 118 (1996), no. 6, 1153-1196. MR 97k:35033

13. L. Capogna and Q. Han, Pointwise Schauder estimates for second order linear equations in Carnot groups, preprint.

14. G. Citti, N. Garofalo, and E. Lanconelli, Harnack's inequality for sum of squares of vector fields plus a potential, Amer. J. Math. 115 (1993), 699-734. MR 94m:35069

15. G. Citti, E. Lanconelli, and A. Montanari, Smoothness of Lipschitz continuous graphs with nonvanishing Levi curvature, Acta Math. 188 (2002), 87-128.

16. G. Citti, M. Manfredini, and A. Sarti, A note on the Mumford-Shah functional in Heisenberg space, preprint.

17. G. B. Folland, Subelliptic estimates and function spaces on nilpotent Lie groups, Ark. Mat. 13 (1975), 161-207. MR 58:13215 
18. B. Franchi, G. Lu, and R. L. Wheeden, Weighted Poincaré inequalities for Hörmander vector fields and local regularity for a class of degenerate elliptic equations, Potential Anal. 4 (1995), 361-375. MR 97e:35018

19. A. Friedman, Partial differential equations of parabolic type, Prentice-Hall, Inc., Englewood Cliffs, NJ, 1964. MR 31:6062

20. P. Hajlasz and P. Koskela, Sobolev met Poincaré, Mem. Amer. Math. Soc. 145 (2000), no. 688. MR 2000j: 46063

21. L. Hörmander, Hypoelliptic second order differential equations, Acta Math. 119 (1967), 147171. MR 36:5526]

22. G. Huisken and W. Klingenberg, Flow of real hypersurfaces by the trace of the Levi form, Math. Res. Lett. 6 (1999), 645-661. MR 2001f:53141

23. D. Jerison and J. M. Lee, The Yamabe problem on $C R$ manifolds, J. Differential Geom. 25 (1987), 167-197. MR 88i:58162

24. D. Jerison and A. Sánchez-Calle, Estimates for the heat kernel for a sum of squares of vector fields, Indiana Univ. Math. J. 35 (1986), no. 4, 835-854. MR 88c:58064

25. H. Kalf, On E. E. Levi's method of constructing a fundamental solution for second-order elliptic equations, Rend. Circ. Mat. Palermo (2) 41 (1992), no. 2, 251-294. MR 93m:35054

26. S. Kusuoka and D. Stroock, Applications of the Malliavin calculus III, J. Fac. Sci. Univ. Tokyo, Sect. IA, Math. 34 (1987), 391-442. MR 89c:60093

27. S. Kusuoka and D. Stroock, Long time estimates for the heat kernel associated with a uniformly subelliptic symmetric second order operator, Ann. of Math. (2) 127 (1988), 165-189. MR 89b:35022

28. E. Lanconelli, Non-linear equations on Carnot groups and CR-curvature problems, Proceeding of the Conference "Renato Caccioppoli and Modern Analysis", to appear in Atti Accad. Naz. Lincei Cl. Sci. Fis. Mat. Natur.

29. E. Lanconelli, A. Pascucci, and S. Polidoro, Linear and nonlinear ultraparabolic equations of Kolmogorov type arising in diffusion theory and in finance, Nonlinear Problems in Mathematical Physics and Related Topics, II, in Honor of Professor O. A. Ladyzhenskaya, International Mathematical Series, 2, to appear.

30. E. Lanconelli and A.E. Kogoj, X-elliptic operators and $X$-control distances, Contributions in honor of the memory of Ennio De Giorgi, Ricerche Mat. 49 (2000), suppl., 223-243. MR 2002c:35121

31. G. Lu, Weighted Poincaré and Sobolev inequalities for vector fields satisfying Hörmander's condition and applications, Rev. Mat. Iberoamericana 8 (1992), 367-439. MR 94c:35061

32. G. Lu, Existence and size estimates for the Green's functions of differential operators constructed from degenerate vector fields, Comm. Partial Differential Equations 17 (1992), 12131251. MR 93i:35030

33. G. Lu, On Harnack's inequality for a class of strongly degenerate Schrödinger operators formed by vector fields, Differential Integral Equations 7 (1994), 73-100. MR 95f:35032

34. A. Montanari, Real hypersurfaces evolving by Levi curvature: Smooth regularity of solutions to the parabolic Levi equation, Comm. Partial Differential Equations 26 (2001), 1633-1664. MR 2002i:35110

35. C. Miranda, Partial differential equations of elliptic type, Ergebnisse der Mathematik und ihrer Grenzgebiete, Band 2, Springer-Verlag, New York-Berlin, 1970. MR 44:1924

36. R. Montgomery, A tour of subriemannian geometries, their geodesics and applications, Mathematical Surveys and Monographs 91, American Mathematical Society, Providence, RI, 2002. MR 2002m:53045

37. J. Petitot and Y. Tondut, Vers une neurogéométrie. Fibrations corticales, structures de contact et contours subjectifs modaux, Math. Inform. Sci. Humaines 145 (1999), 5-101. MR 2000j:92007

38. S. Polidoro, On a class of ultraparabolic operators of Kolmogorov-Fokker-Planck type, Matematiche (Catania) 49 (1994), no. 1, 53-105. MR 97a:35133

39. L. P. Rothschild and E. M. Stein, Hypoelliptic differential operators and nilpotent groups, Acta Math. 137 (1976), 247-320. MR 55:9171

40. Z. Slodkowski and G. Tomassini, Weak solutions for the Levi equation and envelope of holomorphy, J. Funct. Anal. 101 (1991), 392-407. MR 93c:32018 
41. E. M. Stein, Harmonic analysis: real-variable methods, orthogonality, and oscillatory integrals, Princeton Mathematical Series 43, Princeton University Press, Princeton, NJ, 1993. MR 95c:42002

42. F. Uguzzoni, A note on a generalized form of the Laplacian and of sub-Laplacians, to appear in Arch. Math. (Basel).

43. N. T. Varopoulos, L. Saloff-Coste, and T. Coulhon, Analysis and geometry on groups, Cambridge Tracts in Mathematics 100, Cambridge University Press, Cambridge, 1992. MR 95f: 43008

44. C. J. Xu, Regularity for quasilinear second-order subelliptic equations, Comm. Pure Appl. Math. 45 (1992), 77-96. MR 93b:35042

Dipartimento di Matematica, Università degli Studi di Bologna, Piazza di Porta S. Donato, 5 - 40126 Bologna, Italy

E-mail address: bonfigli@dm.unibo.it

Dipartimento di Matematica, Università degli Studi di Bologna, Piazza di Porta S. Donato, 5 - 40126 Bologna, Italy

E-mail address: lanconel@dm.unibo.it

Dipartimento di Matematica, Università degli Studi di Bologna, Piazza di Porta S. Donato, 5 - 40126 Bologna, Italy

E-mail address: uguzzoni@dm.unibo.it 